\title{
A computational study of particulate emissions from Old Moor Quarry, UK
}

\author{
G.M.D. Joseph ${ }^{\mathrm{a}}$, I.S. Lowndes ${ }^{\mathrm{a}}$, D.M. Hargreaves ${ }^{\mathrm{a}, *}$ \\ ${ }^{a}$ Faculty of Engineering, University of Nottingham, Nottingham, NG7 2RD, UK
}

\begin{abstract}
This paper presents an evaluation of the performance of a buoyancy-modified $k-\varepsilon$ dust dispersion model for predicting fugitive dust deposition from a series of bench blast events at a surface quarry in the UK. The dust clouds are modelled as volumetric emissions and their subsequent dispersion simulated by coupling the Eulerian solution of the flow-field with stochastic tracking of the particulates in a Lagrangian reference frame. The coefficients of the turbulence model have been modified and source terms have been added to the turbulence transport equations to permit simulation of both adiabatic and diabatic atmospheric stability conditions. These modifications make the model compatible with MoninObukhuv similarity scaling of the atmospheric surface layer. A procedure is implemented to account for the contribution of mesoscale wind direction variability to the lateral spreading of the dust plume. The Monin-Obukhuv scaling parameters have been derived from routine meteorological data recorded during a month-long monitoring campaign conducted at the case study quarry. Dust deposition measurements from a network of Frisbee gauges are used to validate the predictions of the CFD model. Statistical performance metrics, namely the FAC2 (Fraction of values within a factor of 2 of observations), the MG (Geometric Mean), the FB (Fractional Bias) and the NMSE (Normalized Mean Square Error) have been applied to evaluate the degree of uncertainty in the model
\end{abstract}

\footnotetext{
*Corresponding author (Tel: +44 115846 8079)

Email addresses: enxgmj@nottingham.ac.uk (G.M.D. Joseph),

ian.lowndes@nottingham.ac.uk (I.S. Lowndes), david.hargreaves@nottingham.ac.uk (D.M. Hargreaves)
} 
predictions. The dust deposition predictions of the proposed CFD model are compared to those of the UK-ADMS, to demonstrate how the treatment of the terrain in the CFD model improves the accuracy of the deposition predictions. Keywords: particulates, computational fluid dynamics, deposition

\section{Introduction}

2 Conventionally, dispersion modelling has involved the application of Gaussianbased models such as the UK Atmospheric Dispersion Modelling System (UK4 ADMS) and the US Environmental Protection Agency equivalent, AERMOD, 5 to predict the dispersion of fugitive dust plumes from quarry emission sources 6 and to ensure regulatory compliance. However, whilst these models have relatively fast solution times and are able to predict dust dispersion under a range s of meteorological conditions, Gaussian model algorithms offer an over-simplified 9 resolution of the flow-field over complex terrain and are therefore more suited to modelling dispersion of gaseous plumes emitted from elevated sources over 11 gradually undulating terrain. In this regard, Lowndes et al. (2008) concluded 12 that the reliability of conventional Gaussian model predictions is reduced where the entrainment and dispersion of fugitive dust is complicated by in-pit and surrounding topography as well as the dynamic nature of dust emissions.

Furthermore, within a typical quarry, the terrain gradient is likely to exceed the 1:3 limit for reliable application of the complex terrain algorithms in Gausaccuracy of Gaussian models is challenged by complex terrain and they are unable to account for in-pit fugitive dust retention due to these terrain effects. As a result, they significantly over-predict the long-range transport of particulates by as much as $60 \%$. Consequently, the use of Gaussian models to inform the selection and implementation of fugitive dust abatement strategies for compliance with environmental regulations is likely to result in over-design of these abatement systems. As far as the Environmental Agency is concerned, conventional dispersion models are fit for purpose, their over-predictions ensuring that 
quarries consistently operate within a large factor of safety with regard to dust abatement. However, whilst the conservativeness of Gaussian models may be favourable for environmental protection, it is uneconomical for quarry operators. A need therefore arises to develop new dispersion models which can handle complex terrain and, by extension, resolve the internal flow regimes which occur as a result of significant perturbation of the Atmospheric Boundary Layer (ABL) by pit topography. Ultimately, these models will safeguard against considerable over-design of dust abatement systems, thus proving beneficial for quarry productivity and operating costs.

Zanetti (1990) described dispersion modelling as an important intermediate step in the design and implementation of emission reduction and control measures. To this end, a number of Gaussian models with improved algorithms such as UK-ADMS, AERMOD and CALPUFF are approved for use by the UK Environmental Agency to support Environmental Impact Assessments submitted as part of current or future planning and permitting applications for quarry installations (Appleton et al., 2006; Carruthers et al., 2009).

Di Sabatino et al. (2007) noted that due to their widespread use, Gaussian dispersion models have benefited from extensive model validation and standardization of modelling protocols, and allow the user to model the contribution of a large number of emission sources simultaneously for many hours of meteorological data within a short time. Gaussian-based modelling packages include a utility to extract terrain data from digital formats available on national databases, removing the need for extensive surveys of landforms surrounding a surface quarry (CERC Ltd, 2011). Moreover, both UK-ADMS and AERMOD are equipped with meteorological pre-processors which are able to compute atmospheric parameters to characterize the atmospheric boundary layer from routine meteorological data, thereby eliminating the need for sophisticated meteorological instruments to directly measure these variables (Carruthers et al., 2009).

However, it is well known that Gaussian model algorithms suffer from several inherent limitations related to over-simplification of the flow-field. In the case of 
UK-ADMS, the FLOWSTAR algorithm is used to model the flow over complex terrain. This algorithm uses a linearized analytical solution of the momentum and continuity equations and offers a simplified treatment of topography in which the Froude number is used as a critical model parameter in separated flows (CERC Ltd, 2011). The linearization of the flow equations employed in the UK-ADMS complex terrain model algorithm is based on small perturbation theory by Jackson and Hunt (1975) which is restricted to terrain gradients below 1:3. The theory assumes that terrain in-homogeneities produce small perturbations in the flow-field relative to mean flow quantities. However, this assumption is not valid in cases where separation of the flow occurs (Finardi et al., 1997). In the case of surface quarries, the linearized flow model, and hence the complex terrain algorithm, are incompatible with the quarry topography, which produces large perturbations in the atmospheric flow-field.

Additionally, Gaussian models may suffer from inconsistencies among similar model types or different versions of the same model even with the same data set due to intrinsic differences in model algorithms (Hall et al., 2000). For instance, later version of UK-ADMS offer substantially greater terrain resolution capabilities than earlier versions. Equally, the UK-ADMS treatment of complex terrain is vastly different to that of AERMOD (Carruthers et al., 2011). Also, the formulation of the Gaussian equation implies that model accuracy is severely limited at low wind speeds (Holmes and Morawska, 2006). The reliability of Gaussian model approximations is further reduced for near-ground releases because the vertical dispersion of near-ground releases may depart considerably from the Gaussian probability density function (Smith, 1995). Therefore, ElFadel et al. (2009) recommended that UK-ADMS should only be relied upon as a qualitative prediction tool for dispersion over complex terrain.

There are thus compelling arguments to perform CFD model dispersion studies to produce more realistic models of particulate plume dispersion over complex topography. However, there are few studies in the literature that document the results of CFD investigation of the dispersion and deposition of fugitive dust. Furthermore, the pollutant dispersion studies which incorporate complex ter- 
rain effects, such as those by Chatzipanagiotidis and Olivari (1996), Blocken et al. (2008) and Chavez et al. (2011), only considered the neutral stability case wherein the effects of thermal buoyancy are absent. In these studies, the model predictions were typically validated against wind tunnel measurements and there is a scarcity of studies that have attempted to compare numerical model predictions of dispersion with field measurements. In one of the few instances involving field validation, Hong et al. (2011) employed an LES model to simulate the wind field over a test region in South Korea and subsequently used this validated model to predict the dispersion of livestock odour over this area. Their model predictions were found to correlate well with field measurements. In another example, Scargiali et al. (2005) considered the dispersion of chlorine gas over a mountainous, $30 \mathrm{~km}^{2}$ region in Sicily. To include the effects of thermal buoyancy, they introduced modifications to the RANS equations for turbulent kinetic energy and its dissipation rate. They concluded that predicted ground level concentrations were attenuated by the presence of complex terrain downwind.

Often, in contrast to natural topography, quarry excavations are characterized by sharp changes in elevation due to the steep gradients of the extraction benches. To date, only a handful of researchers have addressed the specific challenges to dispersion modelling presented by quarry topography. Under neutral stability conditions, Silvester et al. (2009) demonstrated that more accurate flow-field resolutions and deposition predictions (when compared to UK-ADMS) can be achieved for the near source dispersion of particulates from an open pit quarry by employing a CFD model. A comparison of the predicted particulate deposition patterns generated by the UK-ADMS and CFD models is shown on Figure 1.

Their study concluded that on average, approximately $50 \%$ of emitted particulates were deposited and retained within the pit boundaries. These model predictions correlated well with pit retention values prescribed by UK Environmental regulations. Furthermore, the degree of pit retention was found to depend on the location of the emission source, the direction of the prevailing 


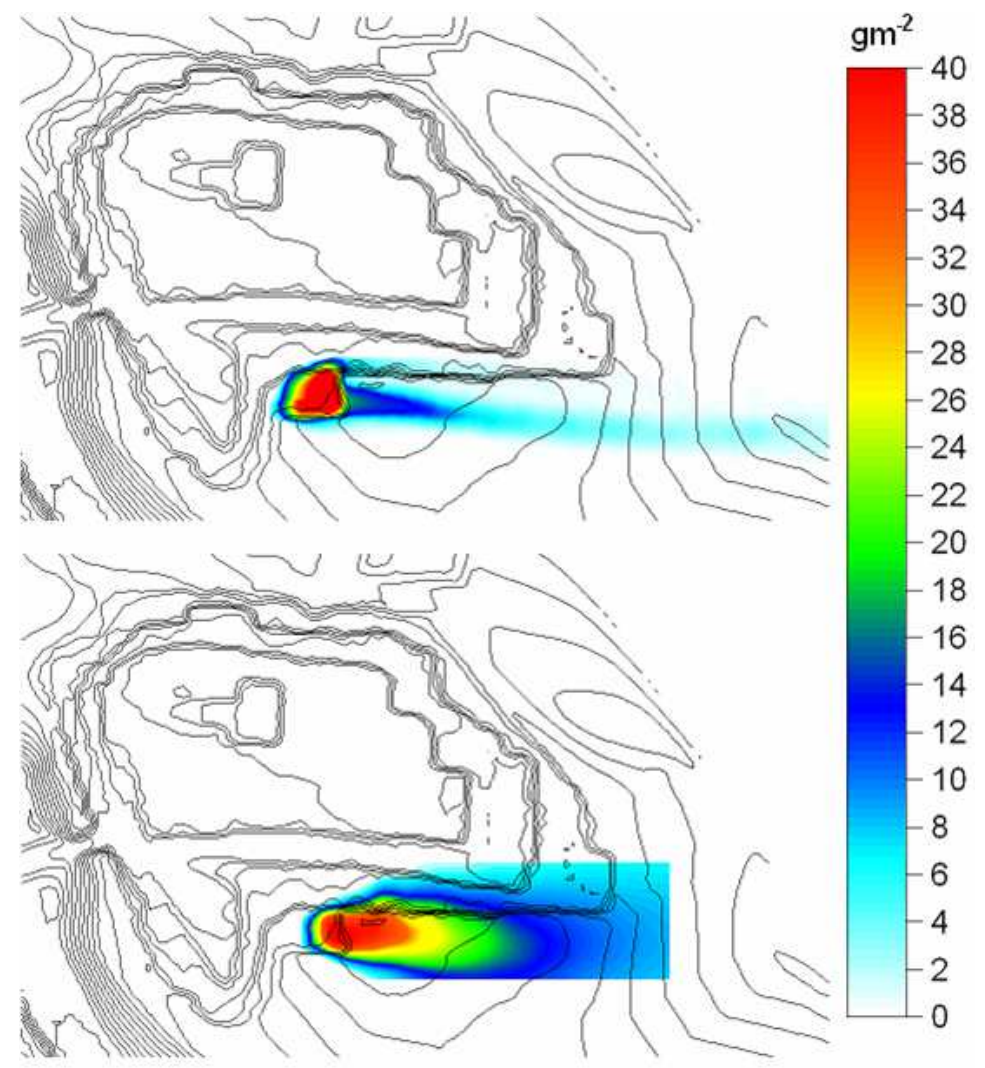

Figure 1: A comparison of the particulate deposition predictions obtained using FLUENT (top) and UK-ADMS (bottom) to model dispersion at a UK quarry under neutral atmospheric conditions from Silvester et al. (2009). 
wind and the nature of the local flow regime generated within the pit cavity.

Chinthala and Khare (2011) have recently used CFD models to investigate the nature of the flow structures which develop in open pit coal mines of varying depth. Their study concluded that as the depth of the pit increased, there was an associated strengthening of the recirculation flows in the pit cavity. The deepest cavities experienced almost complete decoupling of the in-pit flow regime from the atmospheric boundary layer over the open pit and the internal flow was dominated by large vortices. Conversely, for shallower depth pits the penetration of the external atmospheric boundary layer into the pit cavity was more likely to give rise to an internal flow regime dominated by smaller vortices.

Flores et al. (2014) applied a Detached Eddy Simulation (DES) to predict the dispersion of particles injected inside a large open pit copper mine in northern Chile. The depth of the pit was of the same order of magnitude as the height of the daytime ABL. In addition, the pit was situated in a desert region and subject to intense insolation. Flores et al. (2014) anticipated that the recirculations observed by Silvester et al. (2009) would be greatly exacerbated under these conditions, leading to the formation of large scale vortices. Notwithstanding its limitations, Blocken et al. (2008), Tominaga and Stathopoulos (2009) and Chavez et al. (2011) conclude that the standard $k-\varepsilon$ model presents a good compromise between computational demand, results accuracy and model stability. Moreover, Alinot and Masson (2005) used the standard $k-\varepsilon$ model because of the relative ease of deriving the $k$ and $\varepsilon$ turbulence transport properties from routine meteorological data. Furthermore the model equations and coefficients can be readily adapted to make them compatible with Monin-Obukhuv similarity theory, which has been found to adequately characterize the near surface atmosphere.

Silvester et al. (2009) recommended that atmospheric stability conditions should be included in any modelling to obtain more realistic predictions of the atmospheric dispersion of fugitive dust. This paper is the natural extension of the work of Silvester et al. (2009) to include the effects of the varied meteorology encountered during the blasting operations. The computational demand of two 
equation RANS models such as the standard $k-\varepsilon$ turbulence model is relatively low compared to other CFD methods. Consequently, this model may be applied to investigate the effects that different meteorological conditions can have on the dispersion of fugitive dust emitted from multiple bench blasting events.

After a description, in Section 2, of the Old Moor Quarry and its particular meteorology and blasting, a brief description of the UK-ADMS modelling is presented (Section 3). This is then followed by a fuller description of the CFD modelling in Section 4. Then, Section 5 presents results from the CFD modelling and assesses their validity based on a number of performance metrics. Finally, conclusions are drawn from the finding in Section 6 .

\section{Old Moor Quarry}

Old Moor Quarry is located in the Borough of High Peak, Derbyshire and is approximately $4 \mathrm{~km}$ east of the town of Buxton. The Ordnance Survey grid reference for Old Moor Quarry is SK100745 and the quarry is centred on longitude -1.8432 , latitude 53.2653, or Easting 410557 and Northing 374269. At the time of the measurement campaign, the quarry boundaries extended to $835 \mathrm{~m}$ long, $785 \mathrm{~m}$ wide and depth of $69 \mathrm{~m}$.

\subsection{Meteorology}

Hourly-averaged meteorological measurements were collected from a weather station located on site between June $9^{\text {th }}$ to July $19^{\text {th }} 2006$. The meteorological station was operated by the University of Nottingham and the data recorded included: date, time of day, incoming solar radiation $K^{+}$, wind speed at a reference height of $10 \mathrm{~m}, U_{10}$, wind direction, $\theta$, near surface air temperature $T_{a}$, relative humidity, $R_{H}$, and rainfall. The prevailing wind direction for the measurement period was an Easterly wind at approximately $\theta=90^{\circ}$, with average wind speed slightly in excess of $5.1 \mathrm{~m} \mathrm{~s}^{-1}$.

The stability-modified $k$ - $\varepsilon$ model presented in Section 4 is parameterised in terms of the Monin-Obukhuv length, $L$, friction velocity, $u_{*}$, wall temperature, 
${ }_{181}$ the surface,

and surface sensible heat flux,

$$
Q^{*}=Q_{w}+\lambda E+G,
$$

183

$T_{w}$, surface sensible heat flux $Q_{w}$ and aerodynamic roughness height $z_{0}$. In order to derive the Monin-Obukhov length, a number of steps are required. We start with the equations of Holtslag and Van Ulden (1983), for net radiation at

$$
Q^{*}=\frac{(1-a) K^{+}+c_{1} T_{a}^{6}-\sigma T_{a}^{4}+c_{2} N}{1+c_{3}},
$$

where $c_{1}$ is an empirical constant determined by Swinbank (1963) to be $5.31 \times$ $10^{-13} \mathrm{~W} \mathrm{~m}^{-2} \mathrm{~K}^{-6}, T_{a}$ is the near surface ambient air temperature in Kelvin, $c_{2}$ is a constant radiation flux of $60 \mathrm{~W} \mathrm{~m}^{-2}$ which represents the contribution of cloud cover to incoming long-wave radiation in the mid-latitudes and $N$ is the BruntVäisälä buoyancy frequency. $c_{3}$ is a surface heating coefficient, estimated by Holtslag and Van Ulden (1983) to be 0.12. $G$ is the ground heat flux representing energy absorbed by the surface via conduction. Finally, $\lambda E$ denotes the energy required to drive evaporation at the surface and, following the UK-ADMS model, a simplification of the Penman-Monteith equation (Holtslag and Van Ulden, 1983) is used

$$
\lambda E=\frac{\alpha_{P T}}{1+(\gamma / s)}\left(Q^{*}-G\right)+\alpha_{P T} \beta^{\prime} .
$$

where $\alpha_{P T}$ is the Priestley-Taylor evaporation parameter. For the range of atmospheric conditions studied found during the experimental campaign at Old Moor quarry, an intermediate value between $\alpha_{P T}=1.12$ for short grass and $\alpha_{P T}=1.26$ for strongly advective conditions is used (Flint and Childs, 1991). This is considered to be a reasonable estimate of the Priestley-Taylor parameter, since $50 \%$ of the wind observations at the site for the measurement period are greater that $5 \mathrm{~ms}^{-1}$ and a higher value of $\alpha_{P T}$ is recommended by Flint and Childs (1991) to account for increased evaporation from the surface due to high winds.

In Equation 3, $\gamma$ is the psychrometric constant, which is the ratio of the specific heat capacity of water at constant pressure to its latent heat of vaporization, $s$ is the slope of the saturation specific humidity-temperature curve and 


$$
Q_{w}=\frac{\left(1-\alpha_{P T}\right)+(\gamma / s)}{1+(\gamma / s)}\left(Q^{*}-G\right)-\alpha_{P T} \beta^{\prime} .
$$

$\beta^{\prime}$ is a surface moisture constant which is equal to $20 \mathrm{~W} \mathrm{~m}^{-2}$. The ratio $\gamma / s$ decays exponentially with increasing temperature

$$
\gamma / s=\exp \left(0.36-0.056 T_{a}\right) .
$$

The surface sensible heat flux can subsequently be determined from,

Both Holtslag and Van Ulden (1983) and Su (1999) express the ground heat flux $G$ as a fraction of the net radiation $Q^{*}$, which depends on the vegetation cover on the surface,

$$
G=c_{G} Q^{*}
$$

Whilst Holtslag and Van Ulden (1983) apply a constant value of $c_{G}=0.1$, corresponding to a surface covering of short grass, Su (1999) recommends determining the ground cover coefficient by interpolating between the value for dense vegetation canopy and bare soil based on the fractional vegetation cover of the site under consideration,

$$
c_{G}=\Gamma_{c}+\left(1-f_{c}\right)\left(\Gamma_{s}-\Gamma_{c}\right)
$$

where $\Gamma_{c}=0.05$, is the full vegetation canopy coverage coefficient, $f_{c}$ is the fractional canopy coverage and $\Gamma_{s}=0.315$ is the bare soil coefficient. In the UK-ADMS model a fixed value of $c_{G}=0.1$ is used based on the Holtslag and Van Ulden (1983) evaluation that the ground heat flux is generally a small percentage of the net radiation over land surfaces and varying the value of $c_{G}$ between 0.05 and 0.315 has a negligible effect. Hence, we assume a value of 0.1 in the present work.

Once values of surface sensible heat flux and near-surface temperature have been computed from the routine meteorological data, it is possible to estimate the Monin-Obukhuv length using an iterative method which requires approximation of the surface roughness length. For quarry and strip mine operations, USEPA (2008) recommends a surface roughness length of $0.3 \mathrm{~m}$ in AERMET, 
the meteorological pre-processor which accompanies AERMOD. This roughness length accounts for the presence of surface features of the excavation such as benches and slopes. However, since we are including the quarry geometry explicitly here, a roughness length corresponding to the surrounding terrain has been adopted. The quarry is predominantly surrounded by grasslands and low vegetation, thus, a surface roughness length of $z_{0}=0.1 \mathrm{~m}$ has been assumed.

The first iteration is carried out for neutral atmospheric conditions, such that the Businger-Dyer non-dimensional wind shear

$$
\phi_{m}= \begin{cases}\left(1-16 \frac{z}{L}\right)^{-\frac{1}{4}} & -2 \leq z / L \leq 0 \\ 1+\frac{5 z}{L} & 0 \leq z / L \leq 1\end{cases}
$$

has the value of unity, and $u_{*}$ is computed from substitution of the reference wind speed $u_{h}$ into the logarithmic velocity profile equation for the adiabatic atmosphere. The resultant value of $u_{*}$ is used to calculate an initial $L$, hence for the subsequent iterations, corrected values of the non-dimensional wind shear can be determined from the Businger-Dyer functions. Additional iterations are performed until the values of $u_{*}, L$ and $\phi_{m}$ converge.

The Pasquill-Guifford-Turner (PGT) stability classifications are assigned to each line of meteorological data based on the computed values for $L$. This enabled grouping and averaging of the data so that representative meteorology could be computed for each observed stability class. Table 1 lists the average values of the meteorological variables for observations falling under each stability class.

As is typical of diurnal summertime atmospheric conditions in the UK, only four PGT stability classes are required to represent the data contained in Table 1, ranging from class A (strongly unstable) to class D (neutral). The strongly unstable observations appear to be associated with low wind speeds and relatively high values of surface sensible heat flux and near surface temperature. The ABL stability tends towards the neutral case as the wind speed increases and surface sensible heat flux decreases, since more heat is lost to evapo-transpiration processes under these strongly advective conditions. In Figure 2, the frequency 
Table 1: Average values of $L$ corresponding to each PGT stability class for all meteorological data.

\begin{tabular}{lcccc}
\hline PGT class & A & B & C & D \\
\hline$L(\mathrm{~m})$ & -65 & -281 & -1113 & -125415 \\
$Q_{W}\left(\mathrm{Wm}^{-2}\right)$ & 58 & 58 & 24 & 0.4 \\
$T_{W}(\mathrm{~K})$ & 293 & 293 & 289 & 283 \\
$u_{*}\left(\mathrm{~ms}^{-1}\right)$ & 0.351 & 0.570 & 0.680 & 0.799 \\
$\phi_{m}$ & 0.73 & 0.89 & 0.97 & 1.00 \\
$U_{10}\left(\mathrm{~ms}^{-1}\right)$ & 3.5 & 6.1 & 7.4 & 9.2 \\
\hline
\end{tabular}

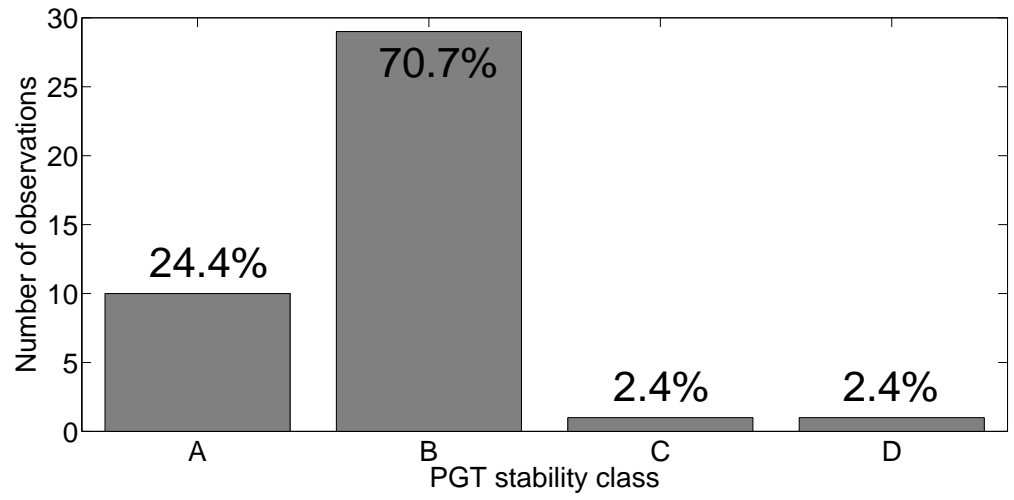

Figure 2: Distribution of PGT stability classes at Old Moor quarry at the time of blasting for the measurement period from June $9^{\text {th }}$ to July $19^{\text {th }}$. 
distribution of stability classes over the observation period at 11:00 hours (blast time) indicate that the site meteorology is largely dominated by unstable atmospheric conditions, with over $90 \%$ of observations falling into either the very unstable or unstable categories (A or B). Average values of $Q_{w}$ and $T_{w}$ have also been calculated for each of the observed stability classes and these have been used to determine corresponding values of $u_{*}$ and $T_{*}$.

\subsection{Characterization of Blasts}

The Michigan Department of Environmental Quality (MDEQ, 2004), provides guidance on the calculation of particulate emission rates from various fugitive dust generating processes within the mineral industries. In the absence of site specific emission factors, generic emission factor estimates compiled by the USEPA AP-42 (USEPA, 1998) for application to fugitive dust emissions from surface coal mining operations in the western United States are recommended for use.

Despite their usefulness, emission factor equations for bench blasting do not account for short-term variability in dust emissions between individual blasts, nor do they account for fluctuations in meteorology or site specific operating conditions. Therefore, they are preferred for estimating continuous releases over relatively long averaging times ranging from one to 24 hours. Consequently, in the present work, the total emission has been modelled as a continuous release occurring over a one hour period.

MDEQ (2004) recommends the use of a fugitive dust emission factor for $\mathrm{PM}_{10}$ of $0.038 \mathrm{~kg}$ per tonne of blasted rock for bench blasting. The studies of Appleton et al. (2006) and Silvester et al. (2006) employ this emission factor to estimate the total suspended particulate emissions from representative blasts recorded at Old Moor quarry. Here, fugitive dust is defined in terms of the inhalable dust fraction consisting of particulates of aerodynamic diameter from $2.5 \mu \mathrm{m}$ to $75 \mu \mathrm{m}$. Since $\mathrm{PM}_{10}$ particulates account for $50 \%$ of the mass of Total Suspended Particulates (TSP) as defined by the size distribution, the MDEQ (2004) estimate is doubled to give an emission factor of $0.076 \mathrm{~kg}$ per tonne for 
total suspended particulates.

By performing an analysis of the video stills recorded during a single blast, Silvester et al. (2006) determined that the dust cloud generated by a blast could be approximated by a cuboid of length $100 \mathrm{~m}$, width $60 \mathrm{~m}$ and height equal to that of the bench. A volumetric emission source consisting of uniformly distributed points was used to define an injection source for the Lagrangian particle tracking model which tracks the particles in the domain. This method of seeding particles is continued in this work and a volumetric source with TSP injection points at $5 \mathrm{~m}$ spacing throughout a cuboid of dimension corresponding to that of the dust cloud has been defined in the Lagrangian particle tracking model (Section 4). The same blast dimensions have been assumed throughout in order to simplify the model set-up, on the basis that a similar configuration of explosive charges was used for all of the blast events monitored at the Old Moor Quarry.

In Table 2, the Easting and Northing coordinates of the centres of the blasts have been obtained from the blast logs and converted to Cartesian coordinates relative to an origin positioned at Eastings 410557, Northings 374269 and $z=$ $-37.00 \mathrm{~m}$. The average emission rate, $m_{\mathrm{avg}}$, associated with each blast is also given in the table.

The source regions are illustrated in Figure 3(a) and it can be seen that some are very close to each other. Therefore, for expediency, the blasts clouds in these clusters are represented by "average" blasts as shown in Figure 3(b). The bounding vertices and average emission rates of these average blasts are listed in Table 3.

\subsection{Frisbee Gauge Measurements}

A Minerals Industry Sustainable Technology (MIST) funded dust monitoring campaign described by Lowndes et al. (2008) was conducted several years prior to this study to provide dust deposition data for the validation of quarry dust dispersion models. The campaign consisted of the installation of a network of Frisbee gauges at locations outside the south-eastern perimeter of the Old 
Table 2: Coordinates of the centre point of the bench faces.

\begin{tabular}{cccccccc}
\hline Location & Eastings & Northings & $\begin{array}{c}x \\
(\mathrm{~m})\end{array}$ & $\begin{array}{c}y \\
(\mathrm{~m})\end{array}$ & $\begin{array}{c}z \\
(\mathrm{~m})\end{array}$ & $\begin{array}{c}h_{\text {bench }} \\
(\mathrm{m})\end{array}$ & $\begin{array}{c}\dot{m}_{\text {avg }} \\
\left(\mathrm{kg} \mathrm{s}^{-1}\right)\end{array}$ \\
\hline 1 & 410913 & 373911 & 356 & -358 & -25.625 & 22.75 & 0.0438 \\
2 & 411093 & 373852 & 536 & -417 & -9.25 & 15.50 & 0.3738 \\
3 & 410995 & 373840 & 438 & -429 & -10.25 & 13.50 & 0.2080 \\
4 & 411256 & 373963 & 699 & -306 & -8.00 & 18.00 & 0.2739 \\
5 & 410585 & 373984 & 28 & -285 & -47.50 & 17.00 & 0.2934 \\
6 & 411292 & 373899 & 735 & -370 & -8.1 & 16.20 & 0.4725 \\
7 & 410661 & 373984 & 104 & -285 & -47.75 & 16.50 & 0.4680 \\
8 & 411206 & 374039 & 649 & -230 & -8.00 & 18.00 & 0.3038 \\
9 & 410509 & 373984 & -48 & -285 & -47.75 & 16.50 & 0.3201 \\
10 & 410625 & 373996 & 68 & -273 & -45.45 & 21.10 & 0.4581 \\
11 & 410990 & 374226 & 433 & -43 & -27 & 20.00 & 0.4372 \\
12 & 411169 & 373852 & 612 & -417 & -9.5 & 15.00 & 0.2292 \\
\hline
\end{tabular}

Table 3: Bounding vertices and average emission rate of representative blast calculated from averaging groups of overlapping blast.

\begin{tabular}{cccccccc}
\hline Location & $\begin{array}{c}x_{\min } \\
(\mathrm{m})\end{array}$ & $\begin{array}{c}x_{\max } \\
(\mathrm{m})\end{array}$ & $\begin{array}{c}y_{\min } \\
(\mathrm{m})\end{array}$ & $\begin{array}{c}y_{\max } \\
(\mathrm{m})\end{array}$ & $\begin{array}{c}z_{\min } \\
(\mathrm{m})\end{array}$ & $\begin{array}{c}z_{\max } \\
(\mathrm{m})\end{array}$ & $\begin{array}{c}\dot{m}_{\text {avg }} \\
\left(\mathrm{kgs}^{-1}\right)\end{array}$ \\
\hline $\mathrm{BS} 1$ & 595 & 655 & -420 & -320 & -17.00 & 0.90 & 0.3732 \\
$\mathrm{BS} 2$ & 486 & 568 & -417 & -357 & -17.00 & -1.50 & 0.270 \\
$\mathrm{BE} 1$ & 560 & 620 & -230 & -130 & -17.00 & 1.00 & 0.3038 \\
$\mathrm{CS} 1$ & 306 & 406 & -358 & -298 & -37.00 & -14.25 & 0.0438 \\
$\mathrm{CN} 1$ & 338 & 398 & -103 & -3 & -37.00 & -17.00 & 0.4372 \\
$\mathrm{DS} 1$ & -22 & 78 & -285 & -225 & -56.00 & -39.00 & 0.3849 \\
\hline
\end{tabular}




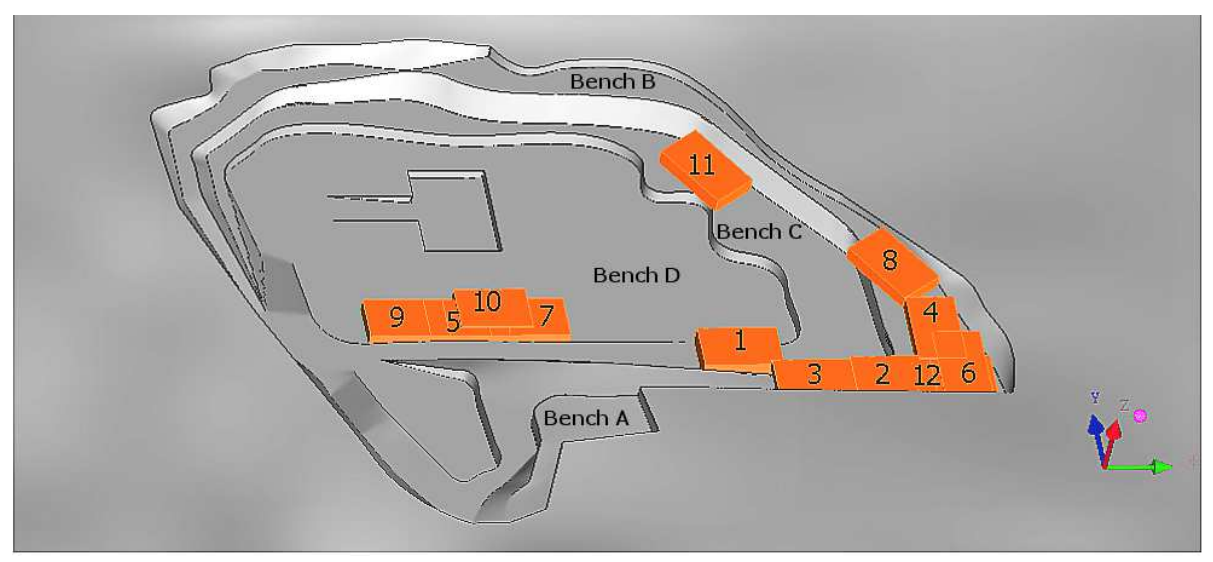

(a)

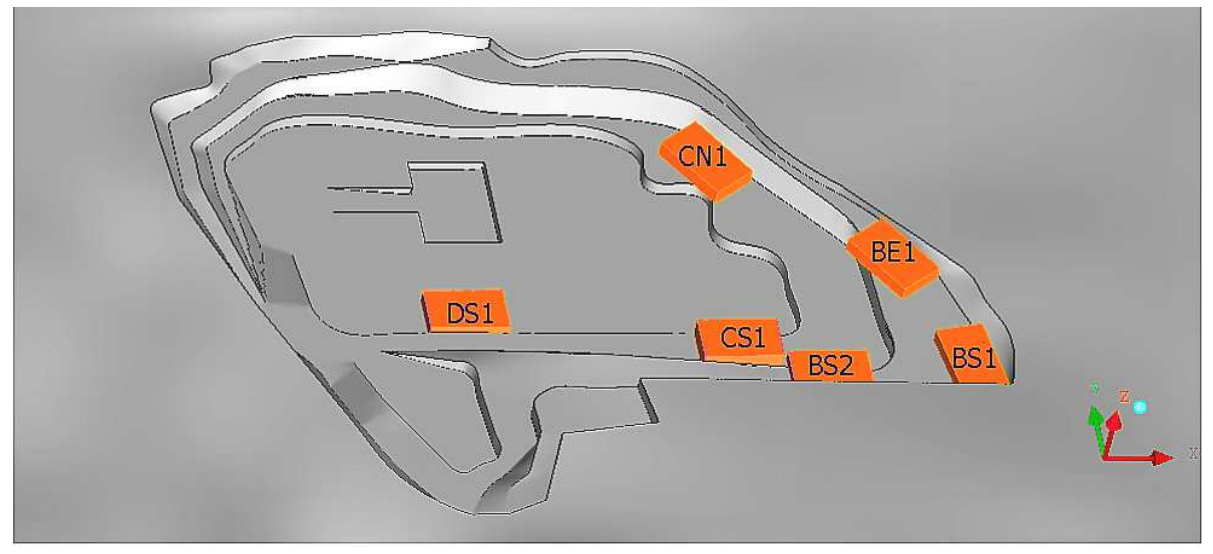

(b)

Figure 3: (a) All initial blast cloud locations for bench blasting conducted during the measurement period and (b) representative blast locations, from averaging groups of blasts in close proximity. 


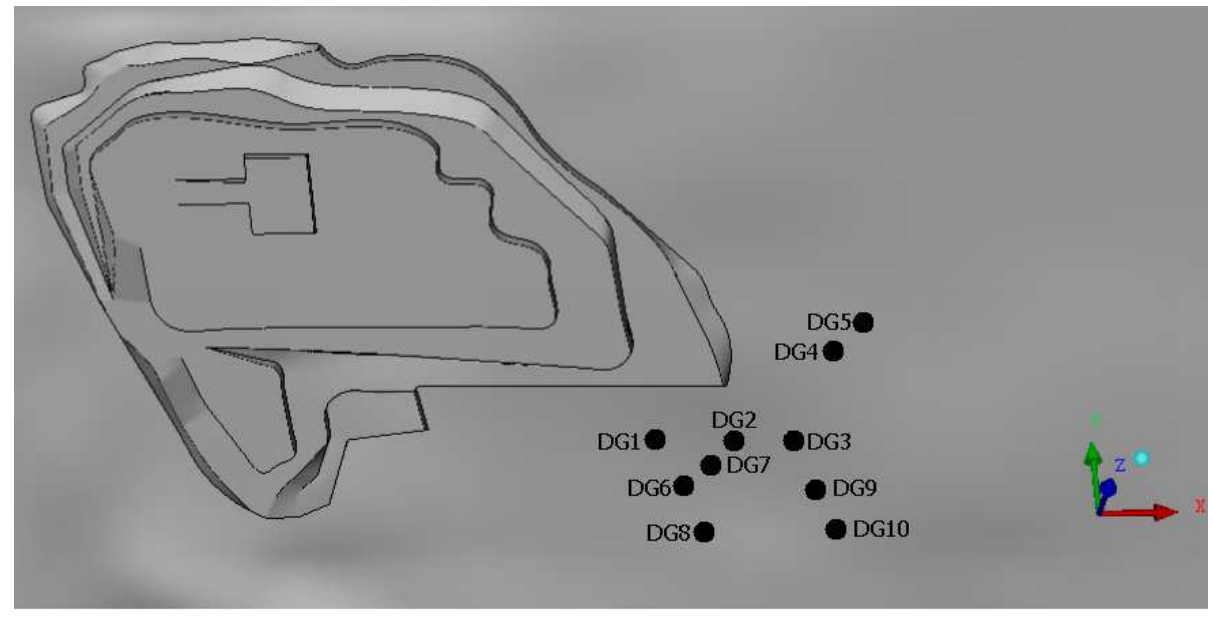

Figure 4: Layout of Frisbee dust gauge positions relative to quarry pit. The gauges are indicated by solid black circles labelled DG1 to DG10.

Moor site. The Frisbee gauges each had an effective collection area, $A_{C}$, of $4.047 \times 10^{-2} \mathrm{~m}^{2}$. The collectors were emptied after each monitoring period of roughly one month and gravimetric analyses were performed using a Malvern Mastersizer $^{T M}$ to determine the mass of dust retained in each gauge.

The blast logs indicated that bench blasting operations were only conducted on 17 days of the monitoring campaign. The duration of a blast event is generally less than $2 \mathrm{~s}$ - from detonation of the explosives to collapse of the bench face and depends on the timing of delay sequences used to detonate the explosive charges.

Figure 4 presents a schematic of the location of Frisbee dust gauges 1 to 10.

\section{UK-ADMS Modelling}

In order to calculate the concentration field of a pollutant plume, the UKADMS atmospheric dispersion model applies the Gaussian plume equation, which is a special solution of the advection-diffusion equation. The Gaussian plume equation is derived under the assumption that steady-state meteorological conditions, in particular - constant wind velocity, persist over the duration 
of the meteorological averaging time. Furthermore, the equation is based on the premise that advection is the dominant mechanism of mass transport in the mean wind direction.

Within the context of the meteorological averaging time, UK-ADMS accounts for complex topography and meteorological variability through modifications to the lateral and vertical plume spread parameters, $\sigma_{y}$ and $\sigma_{z}$; the values of which are dependent on the flow-field computations of the built-in FLOWSTAR complex terrain module. To compute the wind field, FLOWSTAR first constructs a regularly spaced 2D grid which describe the extents of the topographic area specified by the user either through a comma separated variable (CSV) terrain file or Ordnance Survey digital terrain National Transfer Format (NTF) file. Here, a user-defined CSV file has been used to describe the quarry topography. Secondly, the boundaries of the modelling domain are defined by FLOWSTAR using a rectangle aligned with the wind direction. This approach is repeated for each wind direction entered in the meteorological module. The FLOWSTAR algorithm accepts grid densities in the range of $32 \times 32$ to $256 \times 256$ points and produces a Fourier transform which filters out the less significant terrain features, thus capturing the main spatial structure of the terrain. Finally, the Fourier transforms are inverted to determine the flow perturbation velocities, which are subsequently used to adjust the velocity field and ultimately modify the plume spread parameters and height of the plume centreline (Hill et al., 2005; CERC, 2013).

A Stereolithographic (STL) file consisting of triangulated 3D surface geometry describing the topography of Old Moor quarry and its surroundings was used to construct the ground boundary of the CFD computational domain. Thus to ensure consistency between the quarry topography used in the ANSYS Fluent CFD model and that used in the UK-ADMS model, the Cartesian coordinates of the triangle vertices in the STL surface file were exported to a comma delimited ASCII file which could be directly used to generate a terrain file for importing into the UK-ADMS complex terrain utility. In essence, this procedure allows for both models to be furnished with the same topographic information, notwith- 
standing the fact that differences in the resolution of terrain features are bound to arise due to the comparatively explicit treatment of complex terrain in the CFD model.

\section{CFD Modelling}

Two distinct domains were created during the project: the Artificial Terrain and Actual Terrain models. As the names suggest, they differ in the type of terrain around the pit and also in the extent of the domain. The Artificial Terrain consisted of the pit topology at the centre of a $1750 \times 1750 \mathrm{~m}$ horizontal terrain, with the domain extending up to a height of $200 \mathrm{~m}$. The Actual Terrain model consisted of the same pit topology, this time surrounded by actual terrain with the domain extending $3750 \times 3750 \mathrm{~m}$ up to a height of $400 \mathrm{~m}$.

The Artificial Terrain model was used in an extensive testing and sensitivity study during the project and is reported extensively in Joseph (2015). However, the study using it is not reported here for brevity and the Actual Terrain model becomes the focus of this paper.

\subsection{The Computational Domain and Mesh}

The domain included the quarry pit and the surrounding landforms, including the Great Rocks Dale Valley. The surface geometry of the quarry and surrounding landforms extracted from an Stereolithographical (STL) file. The mesh was then created in ICEM CFD ANSYS (2009). The Octree algorithm was then used to discretize the computational domain by creating an initial volume mesh consisting of tetrahedral elements of maximum length $16 \mathrm{~m}$. An inflation layer comprised of prismatic elements was applied at the ground to resolve the flow in the near-wall region. This prism layer was allowed to grow geometrically from a first cell height of $0.6 \mathrm{~m}$ to a maximum prism height of 0.7 times the tetrahedra base width. Within the quarry pit, a maximum surface mesh size of $2 \mathrm{~m}$ was enforced on the bench faces and tetrahedra size was constrained to a maximum of $8 \mathrm{~m}$ by a spherical density region centred on the pit 


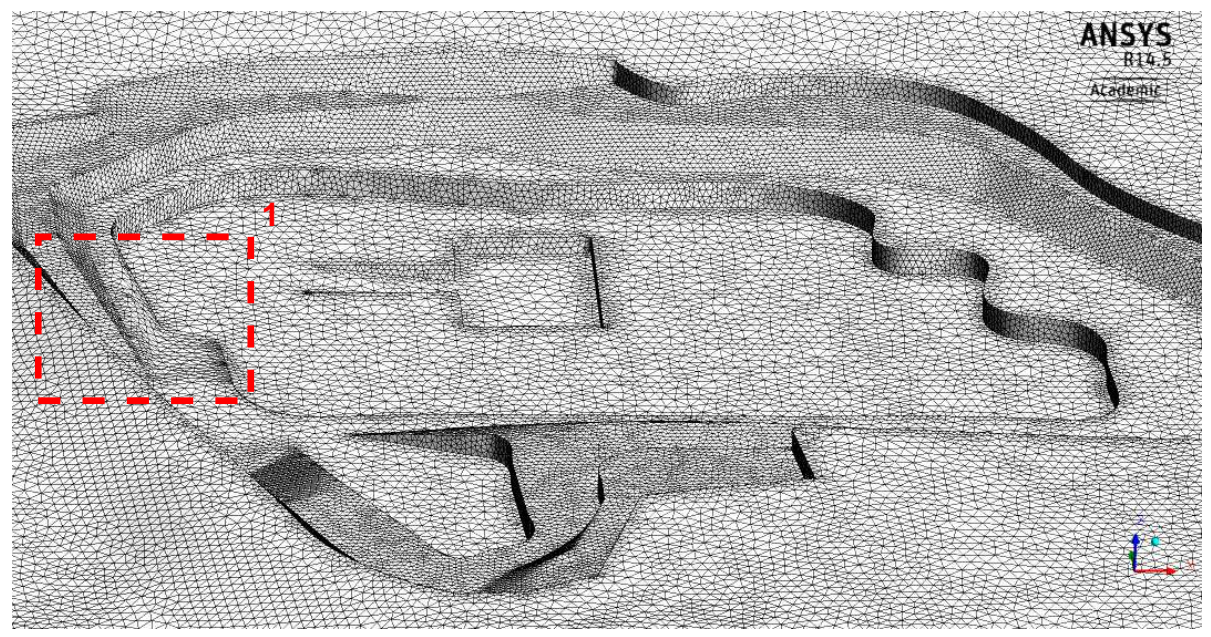

Figure 5: Plan view of surface mesh on the quarry bench floors and bench faces.

and of diameter equal to the maximum pit length. This refinement region was created to capture small-scale features of the in-pit flow. External to the pit, tetrahedra were permitted to grow upward from their interface with the inflation layer towards the top boundary at a growth rate of 1.2 , until the global maximum tetrahedra size of $16 \mathrm{~m}$ was reached.

The mesh was comprised of 18.8 million cells. Simulations using this mesh required distribution of the computations across 2 computed nodes using 16 cores each and 32 GB RAM per node on the University of Nottingham HPC. The run time for each simulation ranged from 8 to 10 hours.

Figure 5 shows the surface mesh including the prismatic boundary layer. The region inside the rectangle is magnified in Figure 6 to better illustrate the prismatic boundary layer applied near the ground.

\subsection{Boundary Conditions}

To accommodate the simulation of multiple wind directions and ensure that the dominant wind component is aligned with the inlet and outlet, the positioning of the pressure outlet is varied. For example, with North being aligned with the $y$-axis in Figure 7 , if the wind direction were within the range $45^{\circ}$ to $135^{\circ}$, 


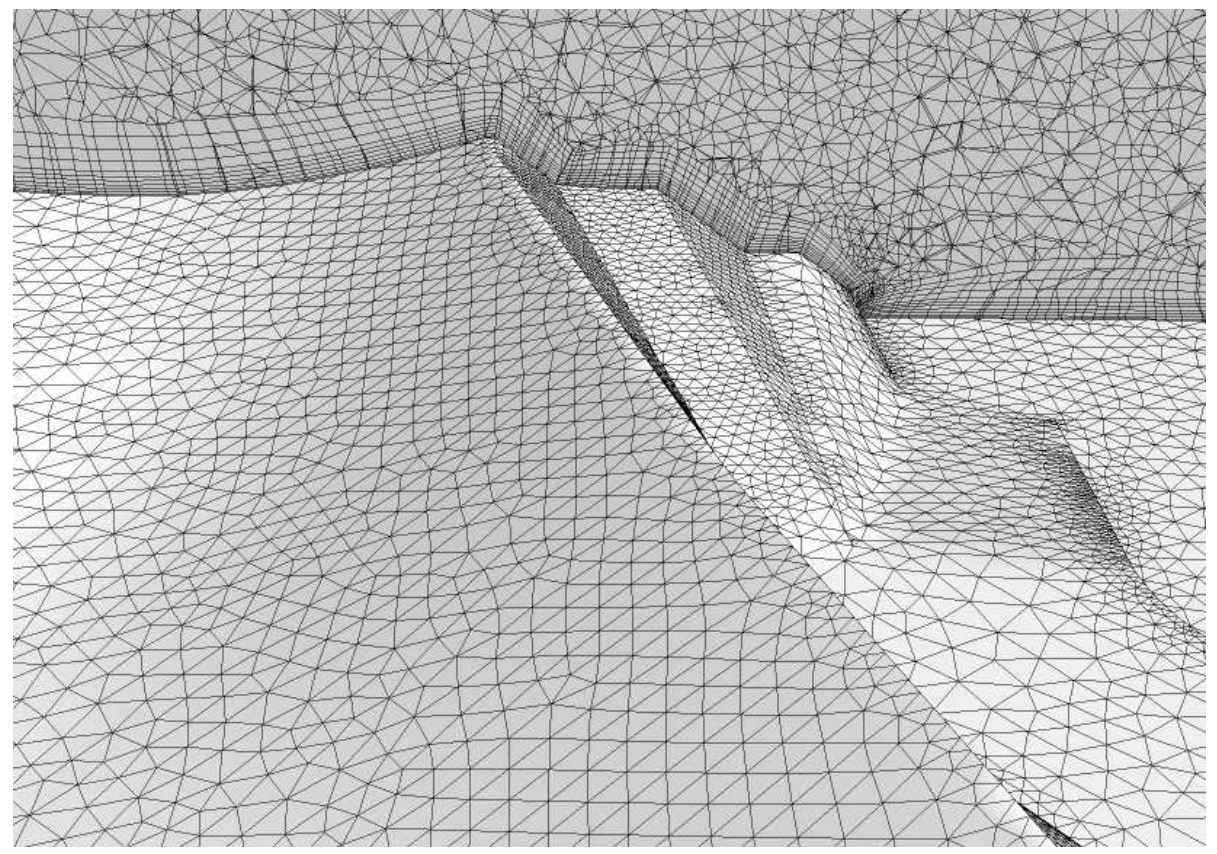

Figure 6: Prismatic layer near the wall in region 1 and cell refinement imposed on benches.

At those inlets, the profiles of the alongwind component of the wind velocity, $u(z)$, the temperature, $T(z)$, the turbulent kinetic energy, $k(z)$ and turbulent dissipation rate, $\varepsilon(z)$ are specified according to the approach of Alinot and Masson (2005). For completeness, we reproduce these profiles here. For $L<0$,

$$
\begin{aligned}
& u(z)=\frac{u_{*}}{\kappa}\left[\ln \left(\frac{z}{z_{0}}\right)+\ln \left(\frac{8 \phi_{m}^{4}}{\left(\phi_{m}+1\right)^{2}\left(\phi_{m}^{2}+1\right)}\right)-\frac{\pi}{2}+2 \tan ^{-1}\left(\frac{1}{\phi_{m}}\right)\right] \\
& T(z)=\frac{T_{*}}{\kappa}\left[\ln \left(\frac{z}{z_{0}}\right)-2 \ln \left[\frac{1}{2}\left(1+\phi_{m}^{-2}\right)\right]\right]-\frac{g}{c_{p}}\left(z-z_{0}\right)+T_{w}
\end{aligned}
$$

and for $L>0$,

$$
\begin{aligned}
u(z) & =\frac{u_{*}}{\kappa}\left[\ln \left(\frac{z}{z_{0}}\right)+\phi_{m}-1\right] \\
T(z) & =\frac{T_{*}}{\kappa}\left[\ln \left(\frac{z}{z_{0}}\right)+\phi_{m}-1\right]-\frac{g}{c_{p}}\left(z-z_{0}\right)+T_{w},
\end{aligned}
$$




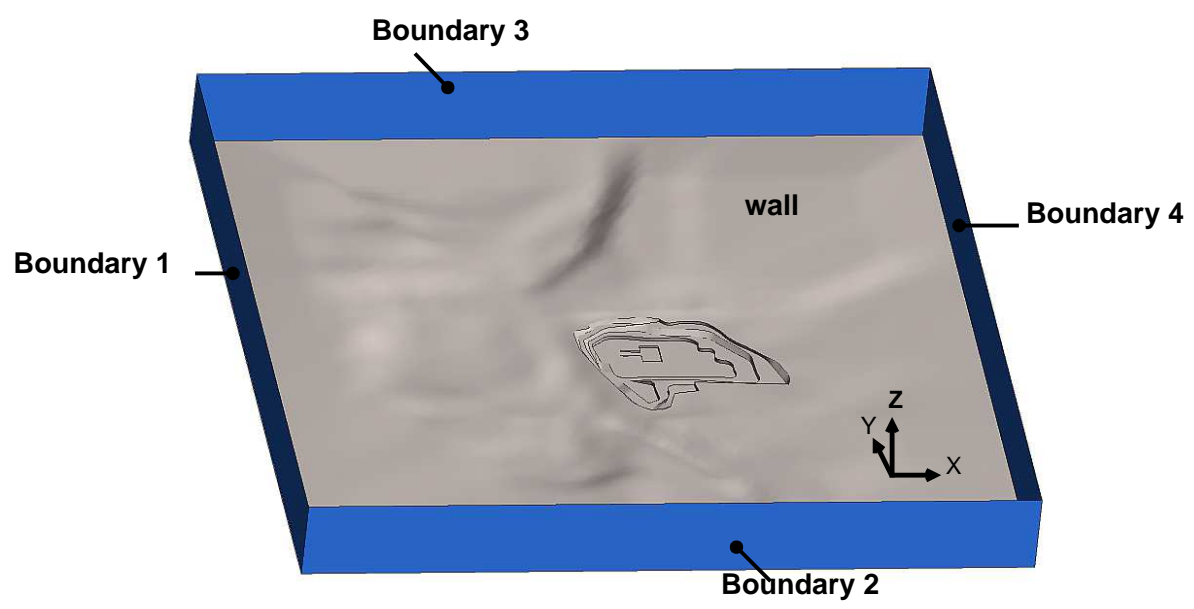

Figure 7: The boundaries of the computational domain used.

410

where $u_{*}$ is the friction velocity and the temperature scale, $T_{*}$, is given by

$$
T_{*}=\frac{-\dot{q}}{\rho c_{p} u_{*}}
$$

where $\dot{q_{w}}$ is the surface heat flux, $c_{p}$ is the specific heat capacity of air, $g$ is the acceleration due to gravity and $\kappa$ is the von Karman constant. The form of the stability similarity function used by Alinot and Masson (2005) are those of Equation 8. The turbulence profiles are

$$
\begin{aligned}
& k(z)=5.48 u_{*}^{2}\left[\frac{\phi_{e}\left(\frac{z}{L}\right)}{\phi_{m}\left(\frac{z}{L}\right)}\right]^{\frac{1}{2}} \\
& \varepsilon(z)=\frac{u_{*}^{3}}{\kappa z} \phi_{e}\left(\frac{z}{L}\right)
\end{aligned}
$$

411 where

$$
\phi_{e}\left(\frac{z}{L}\right)= \begin{cases}1-\frac{z}{L}, & L<0 \\ \phi_{m}\left(\frac{z}{L}\right)-\frac{z}{L}, & L>0 .\end{cases}
$$

412

These boundary profiles were coded into User-Defined Functions (UDFs) for 413 use with ANSYS-Fluent, version 12. With the terrain varying right up to the ${ }_{414}$ boundaries of the domain, $z$ in Equations 9 to 15 had to be modified to prevent 
unphysical behaviour. Thus, $z$ became $z^{\prime}$ where

$$
z^{\prime}=\frac{\partial \psi}{\partial z}+\sqrt{\left(\frac{\partial \psi}{\partial z}\right)^{2}+2 \psi}
$$

and where $\psi$ is the solution to a Poisson equation

$$
\frac{\partial^{2} \psi}{\partial z^{2}}=-1
$$

By using a User-Defined Scalar (UDS) in ANSYS-Fluent and by setting $\psi=0$ on the ground wall, $z^{\prime}$ can be calculated and stored in a User-Defined Memory (UDM) and used in subsequent calculations of the various inlet profiles. In this way, the profiles "hug" the ground surface and negative values of $z^{\prime}$ are impossible. This technique was first proposed by Hargreaves et al. (2006) and Figure 8 shows the modification to the velocity profile in that work.

\subsection{Models}

All simulations were steady-state, Reynolds-Averaged Navier-Stokes (RANS) simulations. In addition to the continuity and momentum equations, the energy equation was modelled and an ideal gas law was used as an equation of state.

Some changes to the standard $k-\varepsilon$ turbulence model were required to reproduce the work of Alinot and Masson (2005). This involved the modification of the model constants and, in particular, the parameter $C_{\varepsilon 3}$ became a function of $z / L$. For reasons of brevity, these modifications are not listed here, but the implementation was tested against the cases quoted in Alinot and Masson (2005) and exact agreement was found.

A Lagrangian particle tracking model (the DPM model in ANSYS-FLuent) was used to model the movement of the dust generated from each of the blasts. As mentioned in Section 2.2, an injection point every $5 \mathrm{~m}$ inside each blast volume was used. Each of these injection points had a mass flow rate equivalent to the total number of particles within the 5 m-sided cube around each injection point. Essentially, each injection was representative of a much greater number of particles. If every particle from the blast were to be tracked, then a solution would not be possible. Particle injection points were horizontally distributed 


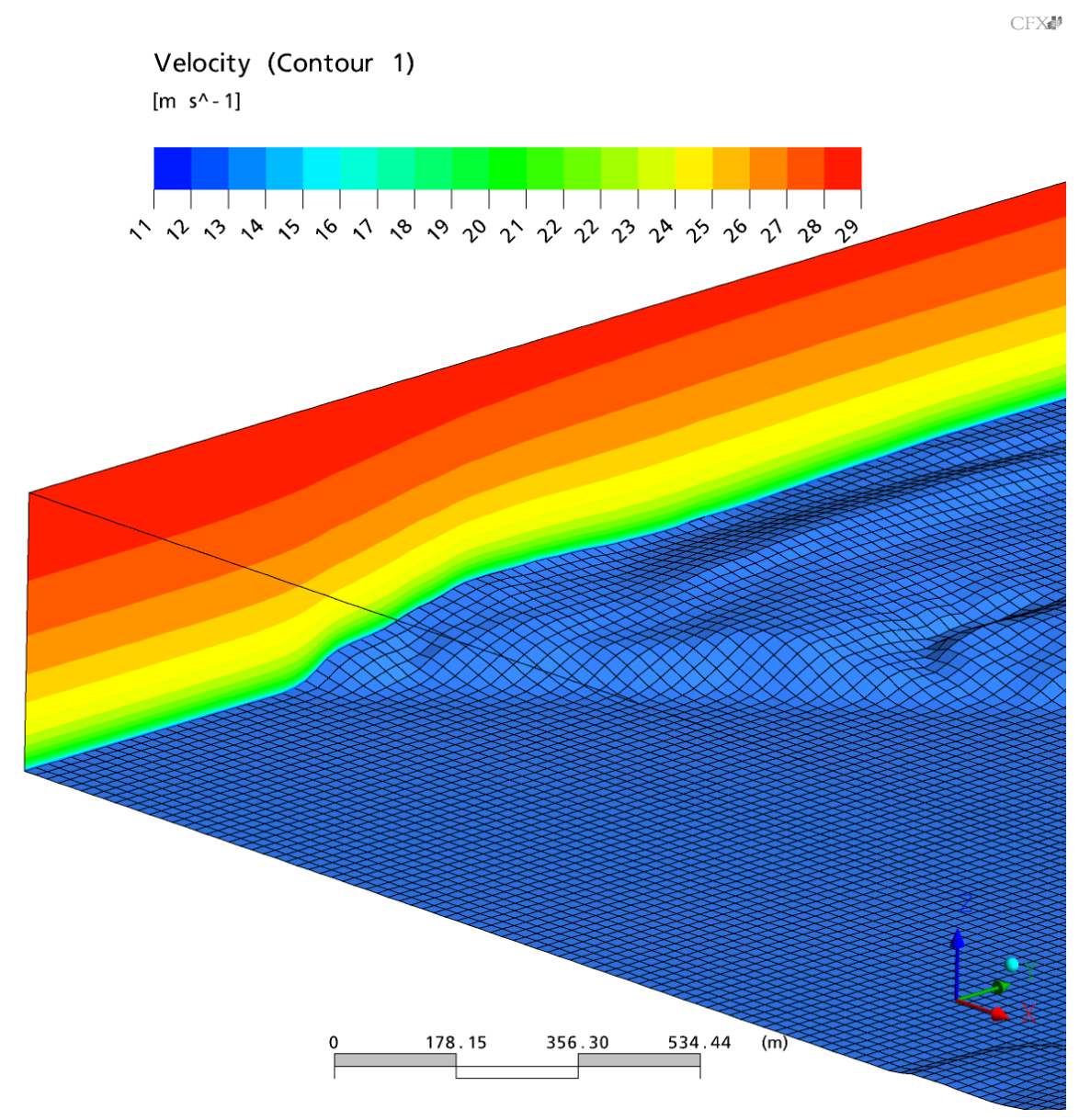

Figure 8: Contours of velocity on an inlet where the topography varies significantly (Hargreaves et al., 2006). 
Table 4: Maximum Particulate aerodynamic diameter of particle size range and the corresponding size fractions which comprise total fugitive dust according to BS6069 part 2:1994.

\begin{tabular}{ccc}
\hline $\begin{array}{c}\text { Dust size }(\mu \mathrm{m}) \\
\text { range }\end{array}$ & $\begin{array}{c}\text { Maximum diameter } \\
\text { of size range }(\mu \mathrm{m})\end{array}$ & Size fraction \\
\hline 30 to 75 & 75 & 0.30 \\
10 to 30 & 30 & 0.20 \\
2.5 to 10 & 10 & 0.45 \\
1.0 to 2.5 & 2.5 & 0.05 \\
\hline
\end{tabular}

${ }_{441}$ at $1.67 \mathrm{~m}$ intervals throughout the representative plan area of the blast cloud.

${ }_{442}$ The injection distribution in the vertical direction along the height of the bench was also $1.67 \mathrm{~m}$. Thus, for the minimum bench height of $13.00 \mathrm{~m}$, a single blast

444 injection was represented using 69120 particles.

445 The studies of Appleton et al. (2006) and Silvester et al. (2009) have adopted ${ }_{446}$ the definition of quarry fugitive dust as consisting of particulates with aerodynamic diameters ranging between 1 to $75 \mu \mathrm{m}$, according to British Standard BS6069 part 2:1994. Mass fractions for particle size ranges which constitute fugitive dust are given in Table 4, from which it may be observed that particles of maximum aerodynamic diameter $\leq 10 \mu \mathrm{m}$ form $50 \%$ of the sampled mass fraction, in accordance with recommendations in the Michigan Department of Environmental Quality air emissions calculation technical report (MDEQ, 2014). Based on this approach, four sizes of particles, corresponding to the maximum value in each range, were injected into the domain at each of the injection loca455 tions.

${ }_{456}$ A number of preliminary tests were made concerning the initial velocity of ${ }_{457}$ the particles at the injection points. It was found that the deposition rates were insensitive to the initial velocity over the likely range of velocities seen

459 in the blasts. As a result, the particles were injected with zero initial velocity.

${ }_{460}$ Physically, it is thought that the particles decelerate quickly due to the drag 
forces acting on them and subsequently move as they are transported in the

462 wind and as they fall under the effects of gravity. Any initial velocity produces

463 a very slight offset from the launch position and nothing more.

\section{Results and Discussion}

\subsection{Flow Field}

The experimental campaign involved no measurements of the external or internal flow fields around the quarry. By inference, however, the dust dispersion validation that is described in Section 5.2 indicates that the flow solver is producing air flows that lead to acceptable dispersion results. This assertion does not automatically follow and it is therefore useful to assess, qualitatively, the flow fields under a variety of conditions. Figures 9 to 12 show contours of

472 the non-dimensionalised along-wind component of velocity,

$$
u_{\theta}=\frac{u \sin \theta+v \cos \theta}{u_{10}},
$$

where $u$ and $v$ are the $x$ and $y$-components of velocity, $\theta$ is the wind direction and $u_{10}$ is the wind speed at a reference height of $10 \mathrm{~m}$ above the ground. Note that North is aligned with the $y$-axis.

In Figure 9 the wind approaches the quarry from the NW and passes over the Great Rocks Dale Valley (seen to the North of the quarry). The wind decelerates as it passes over the valley and this has a bearing on the flow within the quarry. For the Artificial Terrain model, not shown here and which had horizontal terrain around the quarry, a strong recirculation close to the upwind side of the quarry was seen. With the Actual Terrain model shown here, the presence of the valley disrupts the flow and the recirculation zone is not seen

for this wind direction. In the remainder of the figures (Figures 10 to 12) the upwind fetch undulates less and the flow tends to follow the terrain. In all these cases, reverse flow, indicated by the darkest blue contours, is seen, confirming the presence of a recirculation zone on the upstream benches of the quarry. The wind directions shown in the four figures, ranging from the NW to the SW, are 
representative of the prevailing wind directions seen during the experimental

${ }_{489}$ campaign, as is the Pasquill-Gifford Stability Class B. Only the $22^{\text {nd }}$ June case

490 (Figure 11) had a stronger wind with the associated Class D stability.

\subsection{Dust Dispersion}

\subsubsection{Evaluating Model Uncertainty}

Derwent et al. (2010) noted that it is virtually impossible to replicate the full extent of stochastic atmospheric wind systems in a dispersion model, and as such simplifying assumptions are typically adopted to allow the model to simulate the limited range of atmospheric length scales that are most relevant to the turbulent transport processes influencing the dispersion of air pollutants. These simplifications contribute to uncertainty and error in model predictions. Additionally, DEFRA (2009) advise that differences between dispersion model predictions and site measurements are bound to arise in models which rely on the use of emission factor estimates to quantify sources. Approximations of the site meteorology, which are necessary to supply meteorological input parameters that cannot be directly measured at the site, also limit the accuracy of the model. These approximations are not unique to the modified $k-\varepsilon$ model proposed in this work and are routinely used in conventional Gaussian models.

It therefore becomes essential to evaluate the uncertainty in dispersion model predictions through the use of statistical performance metrics which assess how well model predictions correlate with field observations. Ultimately, the current work seeks to establish whether quantifiable gains have been realised in the accuracy of dust dispersion predictions from the quarry using the buoyancy modified $k-\varepsilon$ model. Therefore, evaluation of the $k-\varepsilon$ model uncertainty is conducted in parallel with that of UK-ADMS, to establish a baseline for evaluating the $k-\varepsilon$ model performance.

\subsubsection{Performance Metrics for Dispersion Model Evaluation}

Chang and Hanna (2004) have recommended the use of multiple statistical performance metrics for validation of numerical models because individual met- 


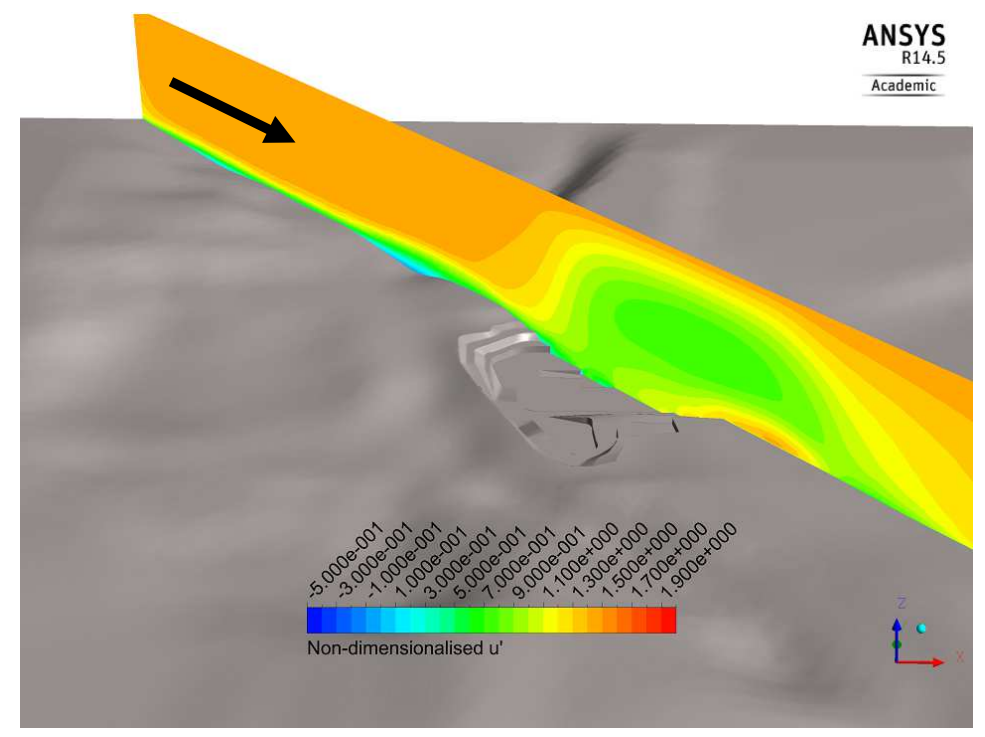

Figure 9: A contour plot of the non-dimensionalized alongwind component of velocity, $u_{\theta}$, on $16^{\text {th }}$ June with $\theta=310^{\circ}, u_{10}=4.5 \mathrm{~m} / \mathrm{s}$ and Class B stability.

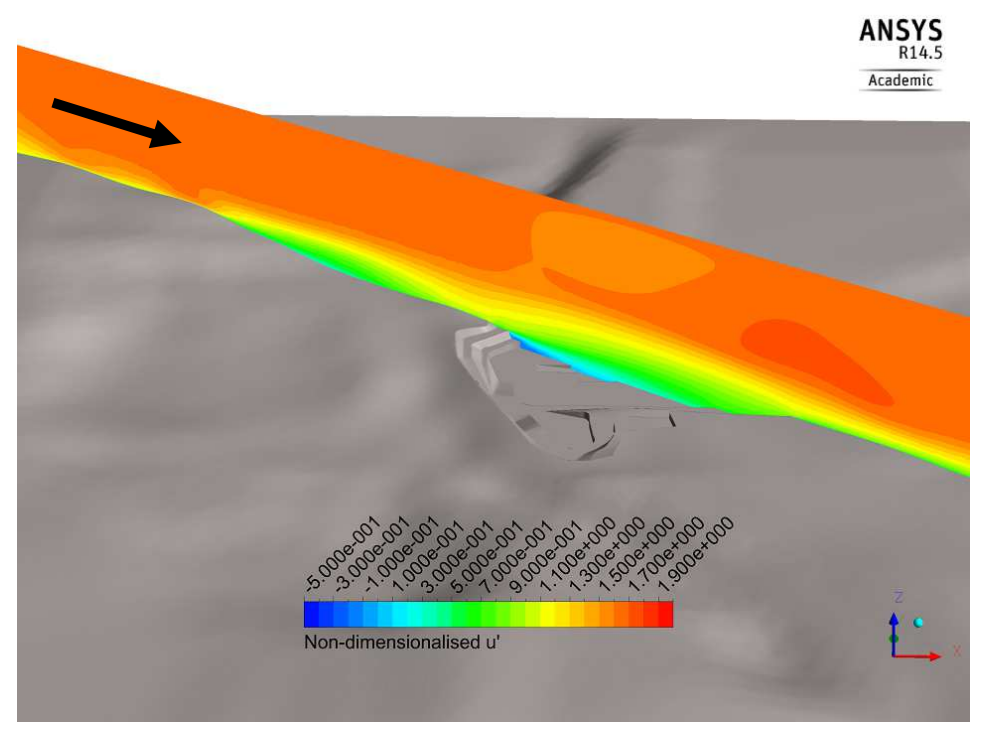

Figure 10: A contour plot of the non-dimensionalized alongwind component of velocity, $u_{\theta}$, on $6^{\text {th }}$ July with $\theta=306^{\circ}, u_{10}=6.2 \mathrm{~m} / \mathrm{s}$ and Class B stability. 


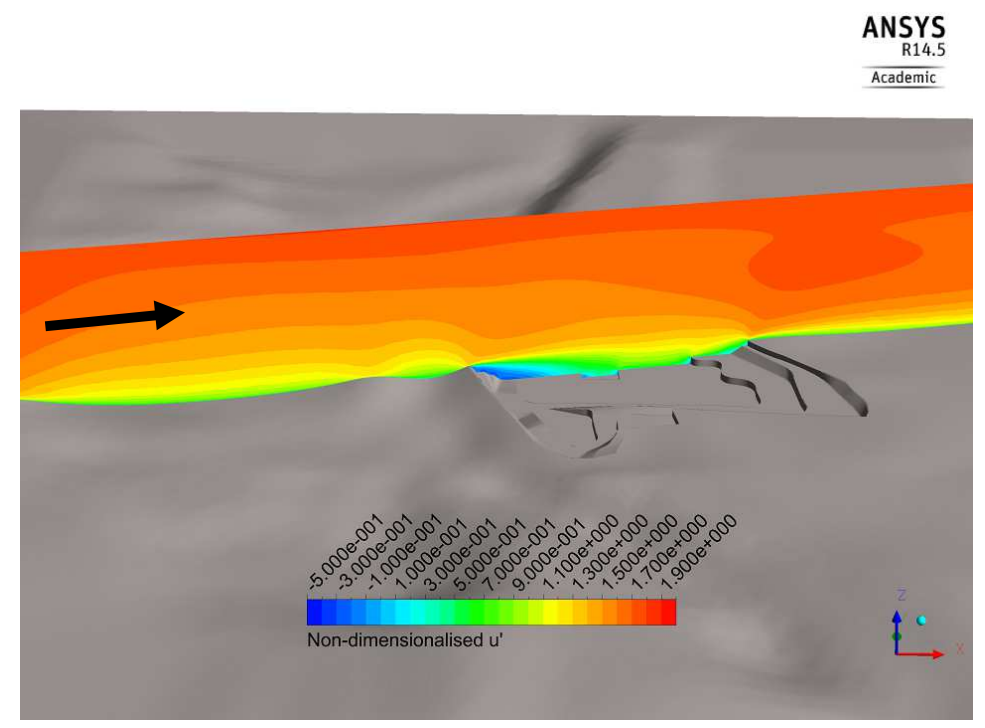

Figure 11: A contour plot of the non-dimensionalized alongwind component of velocity, $u_{\theta}$, on $22^{\text {nd }}$ June with $\theta=258^{\circ}, u_{10}=9.2 \mathrm{~m} / \mathrm{s}$ and Class D stability.

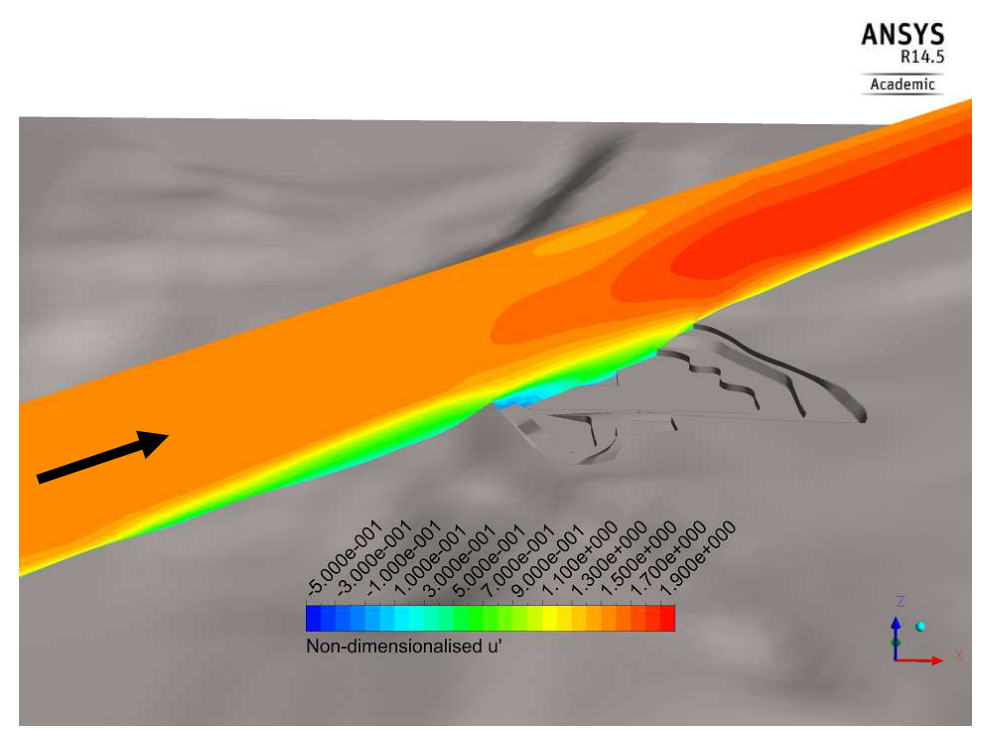

Figure 12: A contour plot of the non-dimensionalized alongwind component of velocity, $u_{\theta}$, on $19^{\text {th }}$ June with $\theta=230^{\circ}, u_{10}=6.0 \mathrm{~m} / \mathrm{s}$ and Class B stability. 
rics are not universally applicable to all dispersion conditions and some may be skewed by outliers. Most dispersion model evaluation studies have made use of the fraction of values within a factor of two of observations, FAC2, which Chang and Hanna (2004); Hanna et al. (2004) describe as the most robust performance metric because it is not overly influenced by outliers. FAC2 is determined from the proportion of the data satisfying,

$$
0.5 \leq \frac{X_{p}}{X_{o}} \leq 2.0
$$

where the subscripts $p$ and $o$ denote predicted and observed values respectively, and $X$, in the context of this study represents the total mass of deposited dust. Besides FAC2, other statistical performance criteria have been selected as recommended by DEFRA (2009). Metrics such as the Fractional Bias, FB, involve normalization of the mean error between model predictions and actual field measurements and are not skewed to favour models that either over-predict or under-predict deposition (Hanna, 1988),

$$
\mathrm{FB}=\frac{\overline{X_{o}}-\overline{X_{p}}}{0.5\left(\overline{X_{o}}+\overline{X_{p}}\right)} .
$$

The geometric mean bias, MG, evaluates the mean error, but on a logarithmic scale. It offers a more balanced treatment of datasets in which individual results vary by several orders of magnitude, however MG is undefined for any zero values which appear in the dataset (Chang and Hanna, 2004),

$$
\mathrm{MG}=\exp \left(\overline{\ln X_{o}}-\overline{\ln X_{p}}\right)
$$

The preceding metrics are useful insofar as quantification of systematic error is concerned. These errors arise from any inaccuracies in the numerical model or dust deposition measuring apparatus and tend to consistently appear across the entire dataset, leaning towards either over-prediction or under-prediction of deposition values. Consequently, another type of performance metric is required to quantify random errors and ascertain the degree of scatter in the data. The normalized mean square error, NMSE, can be used to evaluate uncertainty arising from a combination of systematic and random errors (Hanna et al., 2004; 
Chang and Hanna, 2004),

$$
\mathrm{NMSE}=\frac{\overline{\left(X_{o}-X_{p}\right)^{2}}}{\overline{X_{o} \bar{X}_{p}}} .
$$

543 Further, Chang and Hanna (2004) have derived a relation between NMSE and

544 FB to determine the component of NMSE which is due to systematic errors,

$$
\mathrm{NMSE}_{s}=\frac{4 \mathrm{FB}^{2}}{4-\mathrm{FB}^{2}},
$$
subsequently the random component of the total NMSE, can be obtained from,

$$
\mathrm{NMSE}_{r}=\mathrm{NMSE}-\mathrm{NMSE}_{s} .
$$

where the subscripts $s$ and $r$ refer to systematic and random respectively.

In dispersion studies which attempt to analyse the degree of correlation between the observed and predicted data sets, the correlation coefficient $\mathrm{R}^{2}$ is often computed as a de facto metric for establishing the linear relationship between observed and modelled concentration or deposition. However, Derwent et al. (2010) advise that since $\mathrm{R}^{2}$ may be significantly influenced by outliers in a dataset, it should not be used with small datasets with less than 20 data pairs, where its value is easily distorted by anomalies manifested in one or two data pairs. Therefore, since the current study contains only 10 deposit gauge readings, $\mathrm{R}^{2}$ is not employed as a model performance metric. Hanna et al. (2004) have recommended ranges of the performance metrics for which a numerical dispersion model can be considered suitable for research grade experiments. These include an $\mathrm{FAC} 2>0.5$, which indicates that over $50 \%$ of predictions fall within a factor of 2 of the observations. The mean bias must be within $30 \%$ of the mean such that $-0.3<\mathrm{FB}<0.3$ and $0.7<\mathrm{MG}<1.3$ and a value of $\mathrm{NMSE}_{r}<4$ is considered acceptable for the normalized mean square error component due to random scatter.

\subsubsection{Averaging Time and Wind Direction Variability}

The simulations were set up to account for the hourly-averaged meteorological conditions at the time of each blast event and continuous dust emission rates 
were computed to distribute the mass of liberated dust over the meteorological averaging time. The blast logs also recorded some instances of simultaneously blasting at two benches on the same day and accordingly, dust emissions from both bench locations were modelled in the same simulation. The case study simulations characterize the atmospheric conditions at each blast event using the prevailing wind direction and average meteorological parameters corresponding to the PGT classification observed at the time of the blast event. The total accumulated dust $M_{T}$ for the monitoring period was calculated using the expression,

$$
M_{T}=\sum_{n=1}^{N_{B}} \dot{A} \times T_{\text {exposure }} \times A_{C},
$$

where $T_{\text {exposure }}$ is the time duration of exposure of the gauges to the constant accretion flux $\dot{A}$ predicted for a blast event. $N_{B}$ is the total number of blast events over the monitoring period. Whilst the monitoring period was 41 days, depletion of dust from the ambient air would lead to a reduction in the deposition flux at each receptor location over time. Since the simulations employ a continuous dust emission rate to provide steady-state predictions of the dust accretion, it was deemed necessary to specify an exposure duration over which the constant deposition flux predicted by the the model would be applicable. Therefore the exposure duration was taken as the meteorological averaging time.

As described in Section 3, the UK-ADMS predictions of dry deposition flux were processed in the same way to ensure consistency in the treatment of both sets of predictions.

Table 6 contains observed deposition as well as dust deposition predicted by UK-ADMS and the $k-\varepsilon$ model. The $k-\varepsilon$ predictions consist of two datasets: one for a single simulation at the wind direction stated in Table 5; and one which incorporates a wind direction variability correction.

Vervecken et al. (2013) and Quinn et al. (2001) introduced this approach for CFD modelling to take into account the variation in wind direction during a typical averaging period. Joseph et al. (2014) then generalised the work for all three stability classes, rather than just the neutral case. It is known that the 
wind direction varies around the mean considerably over the kind of averaging periods used in dispersion modelling. Therefore, this is a method which takes into account this variation by conducting a number of CFD simulations at angles centred around the prevailing wind direction, $\bar{\theta}$, from Table 5 .

The observation that the wind direction variability increases with averaging time is represented in empirical formulae by Moore (1976) and also emerges in work by Davies and Thomson (1999) and Mahrt (2010). In both the latter pieces of work it was shown that the standard deviation of wind direction, $\sigma_{\theta}$, remains approximately constant with increasing wind speed above a threshold of $5 \mathrm{~ms}^{-1}$ for both the nocturnal and diurnal ABLs. Joffre and Laurila (1988) proposed characterisation of the wind variability according to the equations,

$$
\sigma_{\theta}(\mathrm{rad})=\left\{\begin{array}{cl}
\frac{0.32}{U_{10}} & U_{10} \leq 5 \mathrm{~ms}^{-1} \\
0.065 & U_{10}>5 \mathrm{~ms}^{-1}
\end{array},\right.
$$

which specify a constant value of $\sigma_{\theta}$ for winds above $5 \mathrm{~ms}^{-1}$. UK-ADMS imposes a limit of $\pm \pi / 6$ to wind direction variability to restrict wind direction variability to realistic values in low wind conditions, thus the component of wind variability due to motions which exceed the turbulence scale is given by:

$$
\sigma_{\theta}=0.065 \sqrt{\frac{7 T_{A}}{U_{10}}}
$$

for $-\pi / 6 \leq \sigma_{\theta} \leq \pi / 6$, where $\sigma_{\theta}$ represents the wind direction variability in radians, $T_{A}$ is the averaging time in hours and $U_{10}$ is the wind velocity in $\mathrm{ms}^{-1}$ at a reference height of $10 \mathrm{~m}$ above the ground (Moore, 1976).

The process of weighting the contribution of each of the directional variations including the mean wind to the resultant plume was automated in MATLAB according to the following equation:

$$
\overline{\dot{A}}=\frac{\sum_{i=1}^{n} p\left(\theta_{i}\right) \dot{A}_{i}}{\sum_{i=1}^{n} p\left(\theta_{i}\right)},
$$

${ }_{616}$ where $\overline{\dot{A}}$ is the weighted average accretion rate, $i$ is an integer corresponding to the simulation number, $n$ is the total number of simulations and $p$ is the probability of occurrence of the $i^{\text {th }}$ wind direction variation. Preliminary work 
(Joseph et al., 2014) revealed that increments of $\sigma_{\theta} / 2$ were sufficient to capture the dispersive effects of wind direction variability. Further, the limits of the variability were taken to be $\pm 3 \sigma_{\theta}$. In all, 13 simulations at angles of

$$
\bar{\theta}-3 \sigma_{\theta}, \bar{\theta}-\frac{5 \sigma_{\theta}}{2}, \ldots, \bar{\theta}, \ldots, \bar{\theta}+\frac{5 \sigma_{\theta}}{2}, \bar{\theta}+3 \sigma_{\theta}
$$

were run for each wind direction. A quadrature method was then used to evaluate the definite integral of the Gaussian function at intervals corresponding to $\sigma_{\theta} / 2$, thus determining the probability of occurrence of each wind direction variation from the following expression,

$$
p\left(\theta_{i}\right)=\frac{1}{\sigma_{\theta} \sqrt{2 \pi}} \int_{\theta_{i}-\sigma_{\theta} / 4}^{\theta_{i}+\sigma_{\theta} / 4} \exp \left[-\frac{(\phi-\bar{\theta})^{2}}{2 \sigma_{\theta}^{2}}\right] \mathrm{d} \phi,
$$

where $\phi$ is the integration variable.

The wind variability post-processing methodology has been applied to obtain weighted summations for the five blast events which contributed most to dust deposition at the gauge locations. Table 5 gives the wind speed, $u_{h}$, the prevailing wind direction, $\bar{\theta}$ and the standard deviation of the wind direction variability, $\sigma_{\theta}$, for each of these blast events.

The observed dust deposition is equivalent to the mass of dust accumulated on the filtration medium. For each of these datasets, a reduction of the emission factor has been considered resulting in two sub-datasets, $\mathrm{EF}_{1.0}$ and $\mathrm{EF}_{0.5}$ which correspond to $100 \%$ and $50 \%$ of the emission factor respectivel (Table 6). Also, the occurrence of zero values in the CFD dataset without wind variability is likely to be due to the use of a finite number of particles injected into the model, since accretion rates at a specific location on the wall boundary are dependent on particles colliding with the wall at that location.

The $k-\varepsilon$ model predictions of cumulative dust deposition over the measurement period have been compared to field observations as well as UK-ADMS predictions. The scatter plots in Figure 13 illustrate, in various forms, the correlation between predicted and observed deposition. DEFRA (2009) recommends that log values of the data also be compared to determine the correlation between predicted and measured values on a logarithmic scale. Normalization of 
Table 5: Values of $u_{h}, \bar{\theta}$ and $\sigma_{\theta}$ for blast events contributing the most to accumulated mass of dust at the gauges.

\begin{tabular}{lrccccc}
\hline $\begin{array}{l}\text { Blast } \\
\text { date }\end{array}$ & & $09 / 06$ & $16 / 06$ & $19 / 06$ & $21 / 06$ & $22 / 06$ \\
\hline$u_{h}$ & $\left(\mathrm{~ms}^{-1}\right)$ & 5.6 & 4.5 & 6.0 & 7.8 & 9.2 \\
$\bar{\theta}$ & $\left(^{\circ}\right)$ & 124.5 & 310.1 & 230.6 & 240.5 & 258.5 \\
$\sigma_{w d}$ & $\left(^{\circ}\right)$ & 5 & 5 & 5 & 5 & 5 \\
\hline
\end{tabular}

Table 6: Predicted dust deposition from CFD and UK-ADMS numerical models compared to site observations of accumulated dust on Frisbee Gauge

\begin{tabular}{ccccccc}
\hline $\begin{array}{c}\text { Gauge } \\
\text { ID }\end{array}$ & \multicolumn{2}{c}{ No wind variability } & \multicolumn{2}{c}{ Wind variability } & UK-ADMS & Observations \\
& $\mathrm{EF}_{1.0}$ & $\mathrm{EF}_{0.5}$ & $\mathrm{EF}_{1.0}$ & $\mathrm{EF}_{0.5}$ & & \\
& $(\mathrm{~g})$ & $(\mathrm{g})$ & $(\mathrm{g})$ & $(\mathrm{g})$ & $(\mathrm{g})$ & $(\mathrm{g})$ \\
\hline FG1 & $1.64 \times 10^{-1}$ & $8.20 \times 10^{-2}$ & $1.68 \times 10^{-1}$ & $8.40 \times 10^{-2}$ & $5.07 \times 10^{-1}$ & $1.36 \times 10^{-1}$ \\
FG2 & $4.12 \times 10^{-2}$ & $2.06 \times 10^{-2}$ & $4.95 \times 10^{-2}$ & $2.48 \times 10^{-2}$ & $3.95 \times 10^{-1}$ & $1.03 \times 10^{-1}$ \\
FG3 & $1.08 \times 10^{-1}$ & $5.39 \times 10^{-2}$ & $5.52 \times 10^{-2}$ & $2.76 \times 10^{-2}$ & $1.55 \times 10^{-1}$ & $1.07 \times 10^{-1}$ \\
FG4 & $4.17 \times 10^{-1}$ & $2.08 \times 10^{-1}$ & $3.01 \times 10^{-1}$ & $1.50 \times 10^{-1}$ & $1.91 \times 10^{-2}$ & $1.10 \times 10^{-1}$ \\
FG5 & $3.33 \times 10^{-1}$ & $1.67 \times 10^{-1}$ & $3.06 \times 10^{-1}$ & $1.53 \times 10^{-3}$ & $4.10 \times 10^{-1}$ & $2.67 \times 10^{-2}$ \\
FG6 & 0.00 & 0.00 & $4.24 \times 10^{-3}$ & $2.12 \times 10^{-2}$ & $3.05 \times 10^{-1}$ & $6.61 \times 10^{-2}$ \\
FG7 & $7.50 \times 10^{-2}$ & $3.75 \times 10^{-2}$ & $7.73 \times 10^{-2}$ & $3.87 \times 10^{-2}$ & $3.10 \times 10^{-1}$ & $6.38 \times 10^{-2}$ \\
FG8 & $7.38 \times 10^{-2}$ & $3.69 \times 10^{-2}$ & $4.16 \times 10^{-2}$ & $2.08 \times 10^{-3}$ & $2.09 \times 10^{-1}$ & $5.97 \times 10^{-2}$ \\
FG9 & $4.51 \times 10^{-2}$ & $2.26 \times 10^{-2}$ & $1.38 \times 10^{-2}$ & $6.88 \times 10^{-3}$ & $8.77 \times 10^{-2}$ & $5.50 \times 10^{-2}$ \\
FG10 & $2.73 \times 10^{-2}$ & $1.37 \times 10^{-2}$ & $7.62 \times 10^{-3}$ & $3.63 \times 10^{-3}$ & $6.40 \times 10^{-2}$ & $5.00 \times 10^{-2}$ \\
\hline
\end{tabular}


the data by either the mean observed or mean predicted deposition is recommended in order to offset systematic errors. In addition, 1:2, 2:1 and 1:1 correlation lines have been superimposed on the plots to permit assessment of $F A C 2$ in accordance with the model performance evaluation procedure prescribed by Derwent et al. (2010).

From Figure 13(a), it appears that UK-ADMS has a tendency to over predict deposition by a factor of 4 . Approximately $60 \%$ of the UK-ADMS predictions are greater than twice the observed deposition and $30 \%$ fall within a factor of 2 of the observations. In contrast, only $20 \%$ of $k-\varepsilon$ model predictions using $100 \%$ emission factor are greater than 2 times the observations and $60 \%$ fall within a factor of 2 of the observations. Out of the $60 \%$ of predictions that were within a factor of 2 of the observations. The wind variability modification reduce both the values and the scatter of the CFD predictions compared to UKADMS. This method is able to smooth out some of the scatter arising from the random fluctuations in individual simulation results. The linear, logarithmic scale and normalized scatter plots all display corresponding trends with regards to the distribution of the data points in each dataset about the 1:1 correlation line. However, the $k-\varepsilon$ model predictions are more evenly distributed about the 1:1 line than those of UK-ADMS.

Figure 14 compares the predicted and observed deposition at each gauge location. According to Barratt (2001), the degree of uncertainty associated with atmospheric dispersion modelling is typically about $50 \%$, however incorrect specification of the input data, such as wind direction and gauge coordinates can produce significant inaccuracies in the model results leading to greater uncertainty, as a result an accuracy up to a factor of two is still considered acceptable for regulatory dispersion models. Error bars have been included in the plot to represent the degree of uncertainty between the predicted and observed data, they range from 0.5 to 2.0 times the observation values.

Figure 14, again shows that UK-ADMS over-predicts the deposition at most of the gauges, registering deposition values well above the top range of the error bars. Both the UK-ADMS and the $k-\varepsilon$ models predict deposition at Gauges 


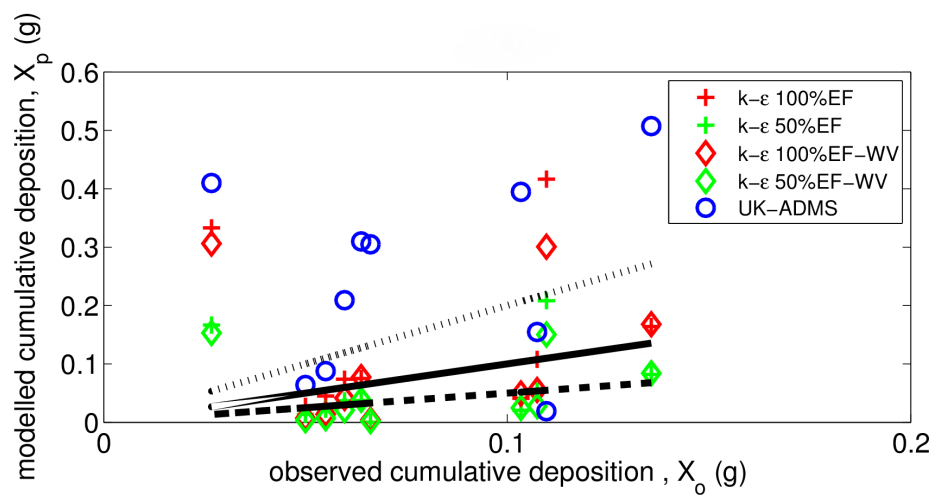

(a)

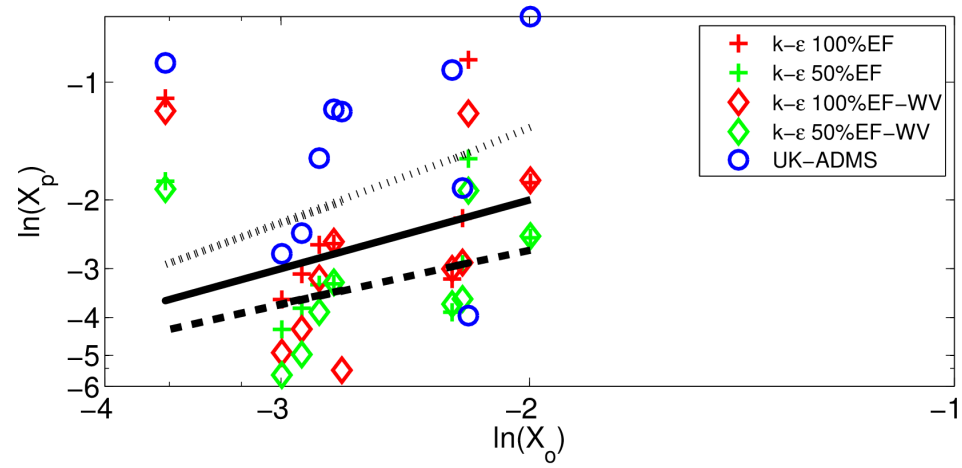

(b)

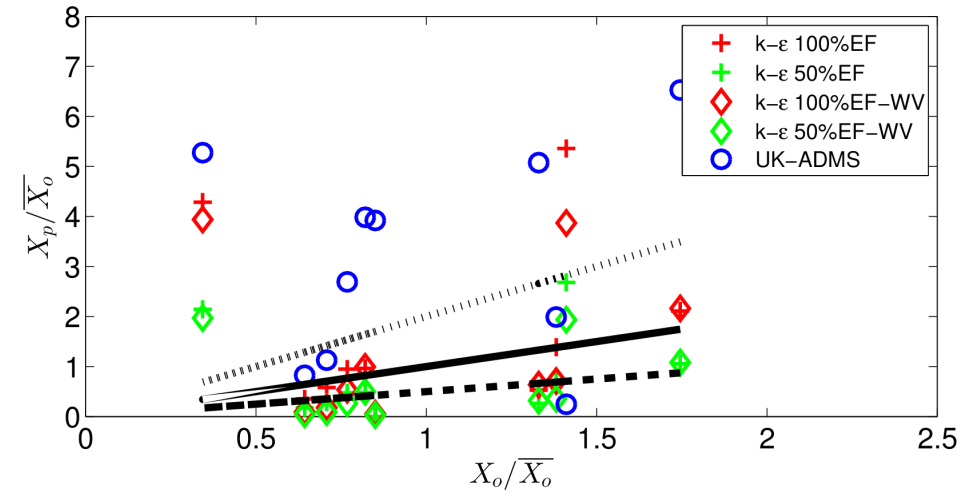

(c)

Figure 13: Predicted dust deposition mass, $X_{p}$, plotted against observed values, $X_{o}$, shown as (a) raw data, (b) raw data on a log-log scale and (c) normalised with respect to the observed data. In each plot, the dotted line represents a correlation of $2: 1$, solid line represents $1: 1$ and dashed line represents $1: 2$. 


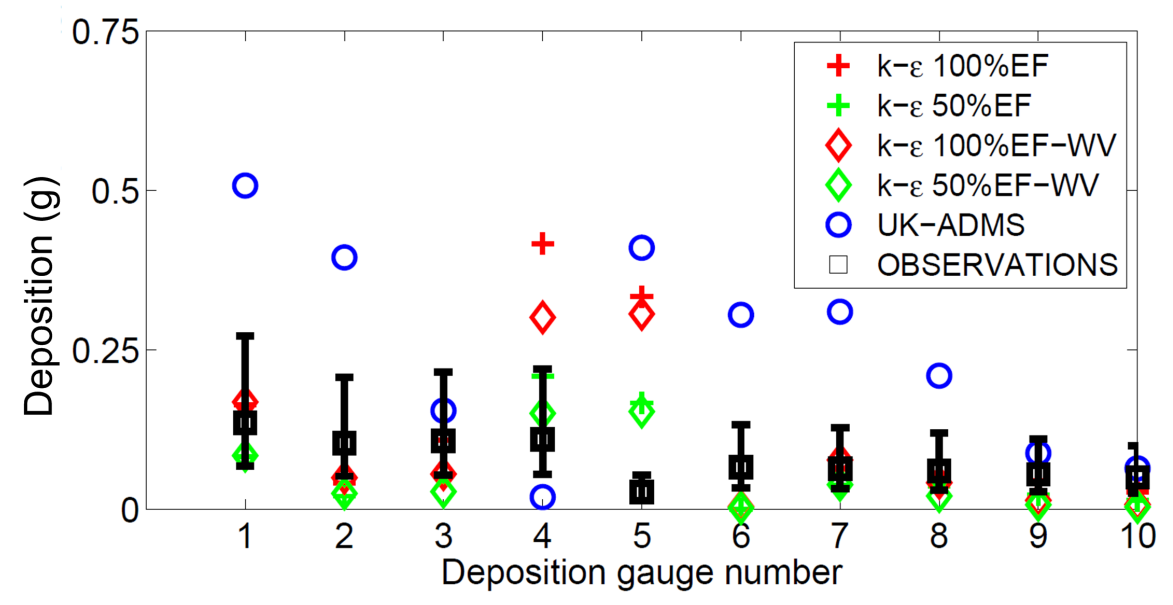

Figure 14: Observed and predicted dust deposition mass at each of the frisbee gauge locations. Error bars on the observed data $(\square)$ are for 0.5 and 2 times the observed value.

4 and 5, which are very inconsistent with the overall deposition trends. ADMS predicts a near zero value of deposition for gauge 4 , whilst the $k=\varepsilon$ predicts the highest deposition both with and without the inclusion of wind variability modifications. In the case of Gauge 5, the observed deposition is the lowest for the entire measured dataset, however both models predict deposition values at this gauge which are one order of magnitude higher than the observation, this result appears to suggest a field measurement error or some local effect that was not captured in either modelling approach. At gauge coordinates further away from the pit boundary, the CFD and UK-ADMS predictions show a greater degree of agreement with the observed deposition.

DEFRA (2009) recommends the use of data conditioning techniques which safeguard against disqualification of otherwise adequate models due to inaccuracies in the input parameters. For short-range model evaluation studies which rely on matching of single data pairs, DEFRA (2009) considers a dispersion model to be suitable for regulatory dispersion modelling applications if the model is capable of predicting the maximum short-term ground level pollutant 


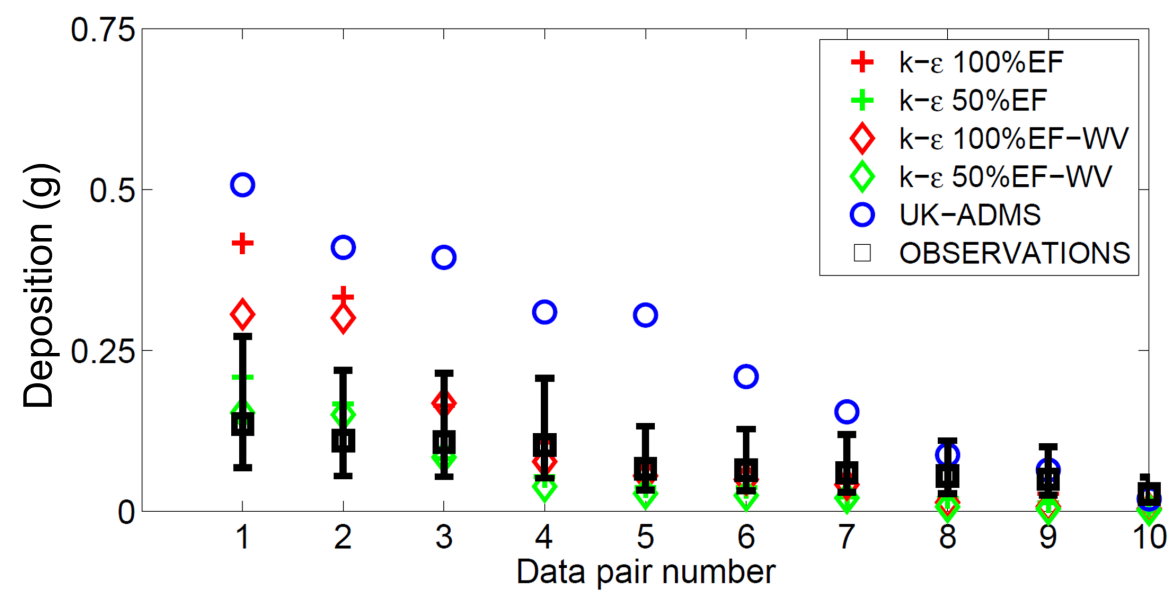

Figure 15: Observed and predicted dust deposition mass for each quantile-quantile data pair. Error bars on the observed data $(\square)$ are for 0.5 and 2 times the observed value.

concentrations at any time or place. The data conditioning techniques endorsed by the US EPA 2003 involve either the arc-maximum or quantile-quantile approach. The arc-maximum technique requires that monitoring stations and the corresponding receptor locations in the modelling domain be configured in a series of concentric arcs at regular distance intervals from the pollutant source. However, the quantile-quantile (Q-Q) technique was considered more appropriate for the gauge configuration used here and seen in Figure 4.

In the Q-Q comparison, the modelled and measured concentrations (or deposition mass) are listed separately in order from largest to smallest: the largest measured values are then paired, followed by the second largest and so on. The concentration pairs are no longer paired in time and space. It is, however, useful in answering the question "Over a period of time and over a variety of locations, does the distribution of model predictions match those of the observations?"(Venkatram, 2000). In this manner, the maximum field observation was compared to the maximum model prediction, as shown in Figure 15.

The performance metrics FAC2, FB, MG and NMSE were computed for both 
Table 7: Statistical Performance metrics computed for CFD, with and without wind variability at $100 \%$ and $50 \%$ of emission factor, and UK-ADMS. Subscript "QQ" denotes performance metrics computed after quantile-quantile conditioning of datasets.

\begin{tabular}{lccccc}
\hline Performance & \multicolumn{2}{l}{ No wind variability } & \multicolumn{2}{c}{ wind variability } & UK-ADMS \\
metric & $\mathrm{EF}_{1.0}$ & $\mathrm{EF}_{0.5}$ & $\mathrm{EF}_{1.0}$ & $\mathrm{EF}_{0.5}$ & \\
\hline FAC2 & 0.60 & 0.50 & 0.50 & 0.30 & 0.40 \\
$\mathrm{FAC} 2_{Q Q}$ & 0.70 & 0.60 & 0.50 & 0.30 & 0.30 \\
$\mathrm{FB}$ & -0.49 & 0.19 & -0.27 & 0.41 & -1.04 \\
$\mathrm{FB}_{Q Q}$ & -0.49 & 0.19 & -0.27 & 0.41 & -1.04 \\
$\mathrm{MG}$ & 0.71 & 1.43 & 1.49 & 2.97 & 0.4 \\
$\mathrm{MG}_{Q Q}$ & 0.71 & 1.43 & 1.49 & 2.97 & 0.4 \\
$\mathrm{NMSE}$ & 1.87 & 0.57 & 1.62 & 1.09 & 2.72 \\
$\mathrm{NMSE}_{Q Q}$ & 1.48 & 0.37 & 0.94 & 0.41 & 2.31 \\
$\mathrm{NMSE}_{s}$ & 0.26 & 0.04 & 0.08 & 0.18 & 1.48 \\
$\mathrm{NMSE}_{s_{Q Q}}$ & 0.26 & 0.04 & 0.08 & 0.18 & 1.48 \\
$\mathrm{NMSE}_{r}$ & 1.73 & 0.96 & 1.55 & 0.92 & 1.24 \\
$\mathrm{NMSE}_{r_{Q Q}}$ & 1.23 & 0.34 & 0.86 & 0.23 & 0.83 \\
\hline
\end{tabular}

the unadjusted and quantile-quantile conditioned datasets (Table 7). An FAC2 of 0.6 was achieved for the $k-\varepsilon$ model predictions without wind variability modifications. The FAC2 improved from 0.6 to 0.7 when the $k-\varepsilon$ predictions without wind variability were adjusted using the quantile-quantile method. UKADMS predictions achieved an FAC2 of 0.4 before data conditioning and 0.3 after, therefore for this study, the FAC2 performance of UK-ADMS was below the recommended minimum of 0.5 . The FAC2 performance of the $k-\varepsilon$ model was marginally better than that of UK-ADMS and within the accepted range, for both the simulations with and without wind variability modifications.

The FB and MG values indicate that the $k-\varepsilon$ model without wind variability modifications over-predicted deposition by a factor of 1.65 when $100 \%$ of the emission factor was considered. When the emission factor was reduced by $50 \%$, 
the model under-predicted by a factor of 1.2. The wind variability modifications improved the correlation of the predictions to the observed deposition when FB was considered. When wind variability was included in the CFD model, the over-prediction decreased to 1.3 for $100 \%$ of the emission factor. At $50 \%$ of the emission factor, the wind variability modified $k-\varepsilon$ model under-predicted the dust deposition by a factor of 1.5 . On the other hand, at $100 \%$ of the emission factor, UK-ADMS over-predicted fugitive dust deposition by a factor of 3.2 ; this over-prediction factor was substantially greater than the corresponding $k-\varepsilon$ predictions. Therefore the $k-\varepsilon$ model out-performs UK-ADMS in terms of the FB. Both models performed poorly for MG, and it is likely that the presence of zero values in the $k-\varepsilon$ predictions without wind variability have affected the reliability of the MG metric.

For $k-\varepsilon$ predictions without wind variability, the values of relative scatter quantified by NMSE were 1.87 and 0.57 for $100 \%$ and $50 \%$ of the emission factor respectively. When the wind variability modifications were included, the NMSE decreased to 1.62 for the full emission factor. At 50\% emission factor, NMSE was 1.09. Conditioning of the data using the quantile-quantile approach re-ordered pairing of the data and the total NMSE improved for all datasets. The component of NMSE due to systematic errors remains unchanged after data conditioning. The relative scatter of the UK-ADMS predictions was substantially greater than all the $k-\varepsilon$ predictions, in particular, the component due to systematic error is nearly 5 times greater than that of the $100 \%$ emission factor $k-\varepsilon$ dataset without wind variability. It may be inferred that such a high systematic error arises due to consistent inadequacies in the resolution of the flow-field by the UK-ADMS model. $\mathrm{NMSE}_{r}$ was greater for the CFD predictions, as this model employs stochastic tracking, however as mentioned previously the wind variability modifications led to a reduction in the random scatter. A considerable improvement was observed in $\mathrm{NMSE}_{r}$, with data conditioning because unlike systematic errors, random errors do not follow any specific trend, nor are they uniformly distributed across the entire data set, hence re-ordering of the data pairs is likely to change the random scatter. 
The results indicate that all the models over-predicted deposition when $100 \%$ of the emission factor was considered. At $50 \%$ of the emission factor, the $k-\varepsilon$ predictions under-predicted the gauge deposition, suggesting that the emission factor equation put forward by MDEQ (2004) over-predicted the emission rate and should be adjusted using a reduction factor between 0.5 and 1 . The $k-\varepsilon$ model outperformed UK-ADMS for all the metrics, and performed satisfactorily for three out of the four metrics. The mean bias was just outside the range recommended for regulatory models, however the model does not over-predict deposition as severely as UK-ADMS, even without wind variability modifications. The performance metrics show that wind variability corrections appear to improve the model performance. Even with such a small data set, the FAC2 results were promising for the $k-\varepsilon$ predictions, and showed a definite improvement over UK-ADMS predictions.

\subsection{Predictions of In-pit Dust Retention}

The tendency of UK-ADMS to under-predict near source dispersion and over-predict long range transport is exemplified in the accretion plots presented in Figure 16. These have been selected for wind directions contributing significantly to dust deposition at the gauges. The $k-\varepsilon$ model predicts peak accretion rates within the pit up to 2 times that of UK-ADMS peak dry deposition predictions. Whilst the overall accretion rate profile is similar for both models, the $k-\varepsilon$ accretion plume appears to be more affected by the terrain than that of UK-ADMS and shows evidence of plume deviation and discontinuities in the accretion profile due to the benches. Further downwind, the accretion plumes decay to achieve similar minima to the UK-ADMS dry deposition plumes, supporting the observation that both models show greater conformity with field observations further away from the perturbed flow regime within and immediately around the pit.

A past study by Silvester et al. (2009) demonstrated that a substantial fraction of the fugitive dust generated within the quarry pit, approximately $50 \%$, is removed near the emission source. Thus, in order to determine whether the CFD 

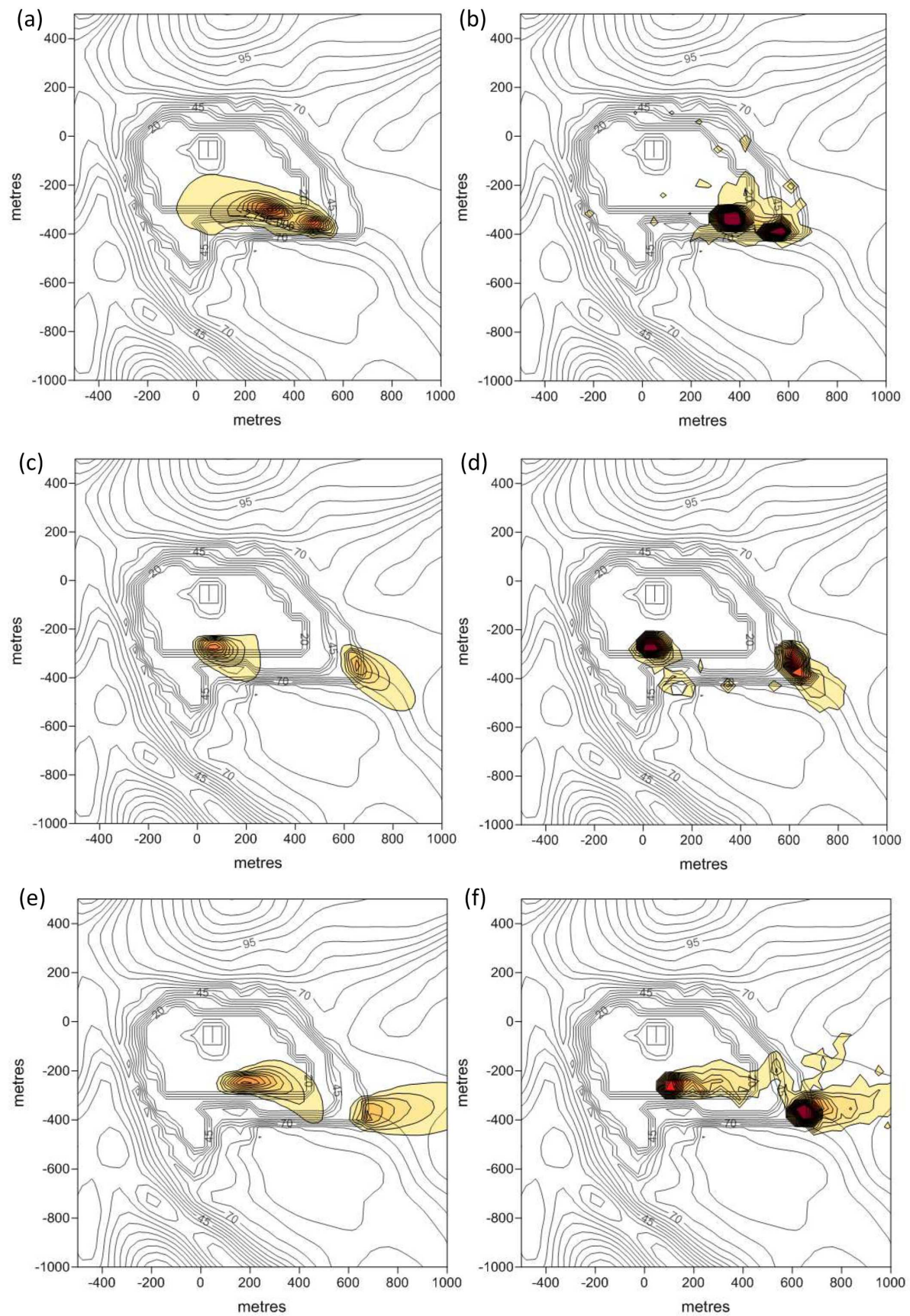

Figure 16: Contour plots of (a),(c),(e) UK-ADMS and CFD (b), (d), (f) deposition rate on (a), (b) $9^{\text {th }}$ June; (c), (d) $16^{\text {th }}$ June; and (e),(f) $22^{\text {nd }}$ June. Dark red corresponds to a deposition rate of $1.625 \times 10^{-5} \mathrm{~kg} \mathrm{~m}^{-2} \mathrm{~s}^{-1}$, while light yellow corresponds to $5.0 \times 10^{-7} \mathrm{~kg} \mathrm{~m}^{-2} \mathrm{~s}^{-1}$. 


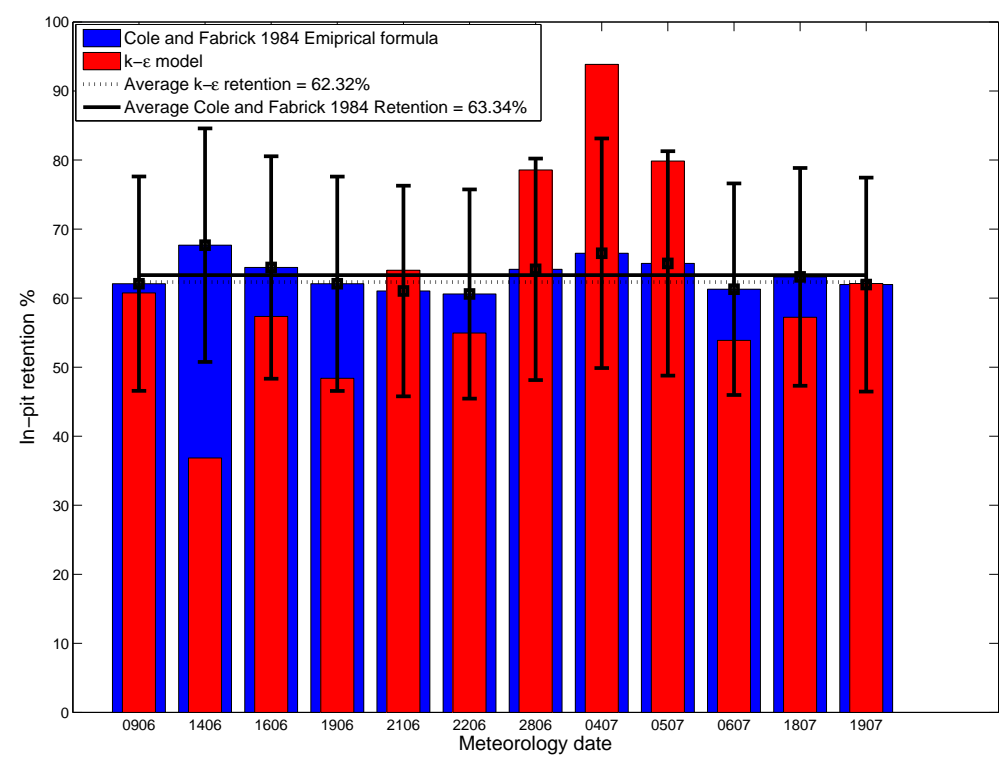

Figure 17: Comparison of the $k-\varepsilon$ model estimates of in-pit retention, to empirical estimates derived from Cole and Fabrick (1984) formula.

794 the empirical estimate of average pit retention. 


\section{Conclusions and Further Work}

The case study commenced with the application of a meteorological preprocessing procedure to derive the requisite model input parameters for MoninObukhuv scaling of the ABL from routine meteorological data, using the formulations of Holtslag and Van Ulden (1983). Subsequently, average meteorological variables were computed to represent the range of stability regimes observed at the site at the time of blasting. $90 \%$ of the meteorological observations were found to fall under the unstable atmospheric classification, which was consistent with the day-time atmospheric conditions expected at the time of blasting.

In the absence of site specific emission data, the USEPA AP-42 Emission factor for bench blasting has been used to estimate fugitive dust emission rates based on the mineral throughput of individual blasts. However, the model performance tests indicate that the emission factor estimates are partially responsible for uncertainty of the predictions, and a reduction factor between 0.5 and 1 is required to compensate for their over-estimation of fugitive dust emissions.

Investigation of the flow structure which developed within the pit revealed that the flow behaviour at the upwind and downwind edges of the pit resembled the flow over backward and forward facing steps respectively. It was seen that external orography had an attenuating effect on the development of recirculation flows within the pit. For instance, when the topography of the Great Rocks Dale valley was included in the computational domain, the backward facing step flow regime did not develop at the entry to the pit for winds perpendicular to the valley axis. Indeed, this appears to suggest that surrounding landforms can potentially disturb the upwind flow and influence the dispersion of dust within and around the quarry.

The model validation exercise formed the crux of the case study, and assessment of the metrics FAC2, FB, MG, and NMSE revealed that the proposed $k-\varepsilon$ model outperformed UK-ADMS in terms of the accuracy of its deposition predictions. The model was able to meet the minimum criteria for the FAC2, MG and NMSE for its predictions without wind variability, using $100 \%$ of the 
emission factor. The predictions which included wind variability averaging were able to satisfy the criteria for FAC2, FB and NMSE. In contrast, UK-ADMS was only able to satisfy the NMSE metric, and even so, the component of NMSE due to systematic errors was about five times that of the $k-\varepsilon$ model without wind variability considerations. Importantly, employing the wind variability post processing methodology reduced the random scatter of the dataset which was likely to be due to the moderating effect of the weighted averaging procedure on random fluctuations of the DPM model.

However, pragmatically speaking, the extra computational expense of CFD simulations for the increase in accuracy seen here, may not be sufficient to persuade practitioners to adopt this approach, except in extreme circumstances. When given the choice between a desktop computer and a significant portion of a compute cluster, the decision to go with the cheaper, Gaussian-based approach is an easy one to make. Further, the use of wind variability imposes an order of magnitude increase in the CFD run times, since at least thirteen simulations are required for a single wind direction.

\section{References}

C. Alinot and C. Masson. $k-\varepsilon$ model for the atmospheric boundary layer under various thermal stratification. Journal of Solar Energy Engineering, 127:438-443, 2005.

ANSYS. FLUENT 12.0 User's Guide. Copyright 2009 ANSYS, Inc., 2009.

T. J. Appleton, S. W. Kingman, I. S. Lowndes, and S. A. Silvester. The development of a modelling strategy for the simulation of fugitive dust emissions from in-pit quarrying activities: a UK case study. International Journal of Mining, Reclamation and Environment, 20(1):57-82, 2006.

R. Barratt. Atmospheric dispersion modelling: an introduction to practical applications. Business and the Environment: Practitioner Series. Earthscan Taylor \& Francis Group, London, UK, 2001. 
B. Blocken, T. Stathopoulos, P. Saathoff, and X. Wang. Numerical evaluation of pollutant dispersion in the built environment: comparisons between models and experiments. Journal of Wind Engineering and Industrial Aerodynamics, 96(1011):1817-1831, 2008.

D. J. Carruthers, C.A. McHugh, E. Vanyyve, M.D. Seaton, and E. Solazzo. Comparison of ADMS and AERMOD meteorological preprocessor and dispersion algorithms, 2009.

D. J. Carruthers, M. D. Seaton, C. A. McHugh, X. Sheng, E. Solazzo, and E. Vanvyve. Comparison of the complex terrain algorithms incorporated into two commonly used local-scale air pollution dispersion models (ADMS and AERMOD) using a hybrid model. Journal of the Air $\&$ Waste Management Association, 61(11):1227-1235, 2011.

CERC. FLOWSTAR complex terrain module. UK-ADMS 5 Technical Specification Documents, Cambridge Environmental Research Consultants, (P14/01Q/13), 2013.

CERC Ltd. FLOWSTAR, Modelling flow over complex terrain, User Guide, 2011.

J. C. Chang and S. R. Hanna. Air quality model performance evaluation. Meteorology and Atmospheric Physics, 87(1-3):167-196, 2004.

A. Chatzipanagiotidis and D. Olivari. Pollutant dispersal downstream of a hill in different wind conditions. Journal of Wind Engineering and Industrial Aerodynamics, 64(23):233-248, 1996.

M. Chavez, B. Hajra, T. Stathopoulos, and A. Bahloul. Near-field pollutant dispersion in the built environment by CFD and wind tunnel simulations. Journal of Wind Engineering and Industrial Aerodynamics, 99(4):330-339, 2011.

S. Chinthala and M. Khare. Particle Dispersion Within a Deep Open Cast Coal Mine, chapter 6. INTECH Open Access Publisher, 2011. 
C.F. Cole and A. J. Fabrick. Surface mine pit retention. Journal of the Air Pollution Control Association, 34(6):674-675, 1984.

B. M. Davies and D. J. Thomson. Comparisons of some parametrizations of wind direction variability with observations. Atmospheric Environment, 33 (29):4909-4917, 1999.

DEFRA. Part IV of the Environmental Act 1995, Environment (Northern Ireland) Order 2002 Part III Local Air Quality Management Technical Guidance LAQM.TG(09), 2009.

D. Derwent, A. Fraser, J. Abbott, M. Jenkins, P. Willis, and T. Murrells. Evaluating the Performance of Air Quality Models. Technical Report Issue 3, DEFRA, www.defra.gov.uk, 2010.

S. Di Sabatino, R. Buccolieri, B. Pulvirenti, and R. Britter. Simulations of pollutant dispersion within idealised urban-type geometries with CFD and integral models. Atmospheric Environment, 41(37):8316-8329, 2007.

M. El-Fadel, L. Abi-Esber, and T. Ayash. Managing emissions from highly industrialized areas: Regulatory compliance under uncertainty. Atmospheric Environment, 43(32):5015-5026, 2009.

S. Finardi, M. Morselli, and P. Jeannet. Wind flow models over complex terrain for dispersion calculations. Technical report, European Cooperation in Science and Technology, 1997.

A. L. Flint and S. W. Childs. Use of the Priestley-Taylor evaporation equation for soil water limited conditions in a small forest clearcut. Agricultural and Forest Meteorology, 56(34):247-260, 1991.

F. Flores, R. Garreaud, and R. C. Muoz. OpenFOAM applied to the CFD simulation of turbulent buoyant atmospheric flows and pollutant dispersion inside large open pit mines under intense insolation. Computers \& F Fluids, 90: 72-87, 2014. 
D.J. Hall, A.M. Spanton, F. Dunkerley, M. Bennett, and R.F. Griffiths. A review of dispersion model intercomparison studies using ISC, R91, AERMOD and ADMS. Technical report, Environmental Technology Centre, Dept. of Chemical Engineering UMIST, 2000.

S. R. Hanna, O. R. Hansen, and S. Dharmavaram. FLACS CFD air quality model performance evaluation with Kit Fox, MUST, Prairie Grass, and EMU observations. Atmospheric Environment, 38(28):4675-4687, 2004.

Steven R. Hanna. Air quality model evaluation and uncertainty. JAPCA, 38 (4):406-412, 1988.

D.M. Hargreaves, T. Porter, and N.G. Wright. Consistent inlet boundary conditions in computational fluid dynamics modelling of wind flow over terrain. In 7th UK Conference on Wind Engineering, pages 47-50, Glasgow, 2006.

R.A. Hill, E.R. Lutman, and A.D. Arnot. Atmospheric dispersion modelling in complex terrain. Technical report, A report prepared for UK Atmospheric Dispersion Modelling Liaison Committee (ADMLC), Westlakes Scientific Consulting, 2005.

N. S. Holmes and L. Morawska. A review of dispersion modelling and its application to the dispersion of particles: An overview of different dispersion models available. Atmospheric Environment, 40(30):5902-5928, 2006.

A. A. M. Holtslag and A. P. Van Ulden. A simple scheme for daytime estimates of the surface fluxes from routine weather data. Journal of Climate and Applied Meteorology, 22(4):517-529, 1983.

S. Hong, I. Lee, H. Hwang, I. Seo, J. Bitog, K. Kwon, J. Song, O. Moon, K. Kim, H. Ko, and S. Chung. CFD modelling of livestock odour dispersion over complex terrain, part ii: Dispersion modelling. Biosystems Engineering, 108(3):265-279, 2011.

P. S. Jackson and J. C. R. Hunt. Turbulent wind flow over a low hill. Quarterly Journal of the Royal Meteorological Society, 101(430):929-955, 1975. 
S. M. Joffre and T. Laurila. Standard deviations of wind speed and direction from observations over a smooth surface. Journal of Applied Meteorology, 27 (5):550-561, 1988 .

G.M.D. Joseph. Improved Dust Dispersion Modelling for Surface Quarries. PhD Thesis, Faculty of Engineering, The University of Nottingham, 2015.

G.M.D. Joseph, D.M. Hargreaves, and I.S. Lowndes. A CFD methodology to incorporate wind direction variability in discrete phase modelling of small particle dispersion. In PHYSMOD 2013 - International Workshop on Physical Modelling of Flow and Dispersion Phenomena, University of Surrey, UK, $16^{\text {th }}$ to $18^{\text {th }}$ September, 2014.

I. S. Lowndes, S. A. Silvester, S. W. Kingman, and D. M. Hargreaves. The application of an improved multi-scale computational modelling techniques to predict fugitive dust dispersion and deposition within and from surface mining operations. In 12 ${ }^{\text {th }}$ U.S./North American Mine Ventilation Symposium, 2008.

L. Mahrt. Surface wind direction variability. Journal of Applied Meteorology and Climatology, 50(1):144-152, 2010.

MDEQ. Calculating air emissions for the Michigan air emissions reporting system (MAERS). Technical report, Clean Air Assistance Program, Environmental Science and Services Division, Michigan Department of Environmental Quality, 2004.

MDEQ. Managing fugitive dust, a guide for compliance with the air regulatory requirements for particulate matter generation. Technical report, Michigan Department of Environmental Quality, 2014.

D. J. Moore. Calculation of ground level concentrations for different sampling periods and source locations(from power plant smokestack). Atmospheric pollution, pages 51-60, 1976.

A. D. Quinn, M. Wilson, A. M. Reynolds, S. B. Couling, and R. P. Hoxey. Modelling the dispersion of aerial pollutants from agricultural buildings an 
evaluation of computational fluid dynamics (CFD). Computers and Electronics in Agriculture, 30(13):219-235, 2001.

F. Scargiali, E. Di Rienzo, M. Ciofalo, F. Grisafi, and A. Brucato. Heavy gas dispersion modelling over a topographically complex mesoscale: A CFD based approach. Process Safety and Environmental Protection, 83(3):242-256, 2005.

S. A. Silvester, I. S. Lowndes, J. Docx, and S. W. Kingman. The application of fluid dynamics to the improved prediction of dust emission from surface quarry operations. In Fifth International Conference on CFD in the Process Industries. CSIRO, 2006.

S. A. Silvester, I. S. Lowndes, and D. M. Hargreaves. A computational study of particulate emissions from an open pit quarry under neutral atmospheric conditions. Atmospheric Environment, 43(40):6415-6424, 2009.

R. J. Smith. A gaussian model for estimating odour emissions from area sources. Mathematical and Computer Modelling, 21(9):23-29, 1995.

Z. Su. The surface energy balance system (SEBS) for estimation of turbulent heat fluxes. Hydrol. Earth Syst. Sci., 6(1):85-100, 1999.

W. C. Swinbank. Long-wave radiation from clear skies. Quarterly Journal of the Royal Meteorological Society, 89(381):339-348, 1963.

Y. Tominaga and T. Stathopoulos. Numerical simulation of dispersion around an isolated cubic building: Comparison of various types of $k-\varepsilon$ models. Atmospheric Environment, 43(20):3200-3210, 2009.

USEPA. AP 42, Fifth Edition, Volume 1 Chapter 11: Mineral products industry. Technical report, U.S. Environmental Protection Agency, 1998.

USEPA. Quality assurance handbook for air pollution measurement systems, 2008. 
989 A. Venkatram. A critique of empirical emission factor models: a case study of the AP-42 model for estimating PM10 emissions from paved roads. Atmospheric Environment, 34(1):1-11, 2000.

${ }_{992}$ L. Vervecken, J. Camps, and J. Meyers. Accounting for wind-direction fluctuations in reynolds-averaged simulation of near-range atmospheric dispersion. Atmospheric Environment, 72:142-150, 2013.

P. Zanetti. Air Pollution Modelling, Theories, Computational Methods and Available Software. Van Nostrand Reinhold, Southampton, Boston, 1990. 